\title{
Ethnobotanical Survey on Bitter Tea in Taiwan
}

\section{OPEN ACCESS}

Edited by: Michael Heinrich,

University College London, United Kingdom

Reviewed by:

Manuel Pardo de Santayana, Autonomous University of Madrid,

Spain

Diego Rivera,

University of Murcia, Spain

Lukasz Luczaj,

University of Rzeszow, Poland

*Correspondence:

Shan-Yu Su

shanyusu@gmail.com

Shyh-Shyun Huang

sshuang@mail.cmu.edu.tw

${ }^{t}$ These authors have contributed equally to this work

Specialty section:

This article was submitted to

Ethnopharmacology,

a section of the journal

Frontiers in Pharmacology

Received: 16 November 2021

Accepted: 13 January 2022

Published: 18 February 2022

Citation:

Chao J, Chen T-Y, Pao L-H, Deng J-S, Cheng $Y-C$, Su S-Y and Huang S-S (2022) Ethnobotanical Survey on Bitter Tea in Taiwan.

Front. Pharmacol. 13:816029. doi: 10.3389/fphar.2022.816029

\author{
Jung Chao ${ }^{1 \dagger}$, Ting-Yang Chen ${ }^{2 \dagger}$, Li-Heng Pao ${ }^{3,4}$, Jeng-Shyan Deng ${ }^{5}$, Yung-Chi Cheng ${ }^{6}$, \\ Shan-Yu Su ${ }^{7 *}$ and Shyh-Shyun Huang ${ }^{5,8 *}$
}

${ }^{1}$ Chinese Medicine Research Center, Department of Chinese Pharmaceutical Sciences and Chinese Medicine Resources, Master Program for Food and Drug Safety, China Medical University, Taichung, Taiwan, ${ }^{2}$ Chinese Medicine Research Center, Department of Chinese Pharmaceutical Sciences and Chinese Medicine Resources, China Medical University, Taichung, Taiwan, ${ }^{3}$ Graduate Institute of Health Industry Technology, Research Center for Food and Cosmetic Safety, and Research Center for Chinese Herbal Medicine, College of Human Ecology, Chang Gung University of Science and Technology, Taoyuan, Taiwan, ${ }^{4}$ Department of Gastroenterology and Hepatology, Chang Gung Memorial Hospital, Taoyuan, Taiwan, ${ }^{5}$ Department of Food Nutrition and Health Biotechnology, Asia University, Taichung, Taiwan, ${ }^{6}$ Department of Pharmacology, Yale University School of Medicine, New Haven, CT, United States, ${ }^{7}$ Department of Chinese Medicine, China Medical University Hospital, School of PostBaccalaureate Chinese Medicine, College of Chinese Medicine, China Medical University, Taichung, Taiwan, ${ }^{8}$ School of Pharmacy, China Medical University, Taichung, Taiwan

Ethnopharmacological evidence: In Taiwan, herbal tea is considered a traditional medicine and has been consumed for hundreds of years. In contrast to regular tea, herbal teas are prepared using plants other than the regular tea plant, Camellia sinensis (L.) Kuntze. Bitter tea (kǔ-chá), a series of herbal teas prepared in response to common diseases in Taiwan, is often made from local Taiwanese plants. However, the raw materials and formulations have been kept secret and verbally passed down by store owners across generations without a fixed recipe, and the constituent plant materials have not been disclosed.

Aim of the study: The aim was to determine the herbal composition of bitter tea sold in Taiwan, which can facilitate further studies on pharmacological applications and conserve cultural resources.

Materials and methods: Interviews were conducted through a semi-structured questionnaire. The surveyed respondents were traditional sellers of traditional herbal tea. The relevant literature was collated for a systematic analysis of the composition, characteristics, and traditional and modern applications of the plant materials used in bitter tea. We also conducted an association analysis of the composition of Taiwanese bitter tea with green herb tea (qing-cao-cha tea), another commonly consumed herbal tea in Taiwan, as well as herbal teas in neighboring areas outside Taiwan.

Results: After visiting a total of 59 stores, we identified 32 bitter tea formulations and 73 plant materials. Asteraceae was the most commonly used family, and most stores used whole plants. According to a network analysis of nine plant materials used in high frequency as drug pairs, Tithonia diversifolia and Ajuga nipponensis were found to be the core plant materials used in Taiwanese bitter tea.

Conclusion: Plant materials used in Taiwanese bitter tea were distinct, with multiple therapeutic functions. Further research is required to clarify their efficacy and mechanisms. 


\section{INTRODUCTION}

Herbal tea is a drink composed of plants other than Camellia sinensis (L.) Kuntze of the Theaceae family-in contrast to regular tea-and is prepared by decocting or brewing with hot water. Herbal tea is commonly prepared with local plants that can be easily obtained (Fu et al., 2018). The custom of brewing tea with herbs is found all over the world, including Europe (Soukand and Kalle, 2013; Soukand et al., 2013), America (Joubert et al., 2008), Africa (Roulette et al., 2018), and Asia (Hu, 2005). The herbs used could be a single herb or a mixture of multiple plants. In many areas, herbal teas are used as therapeutic vehicles to treat associated health conditions (Poswal et al., 2019).

In China, herbal tea has been consumed for more than 2000 years (Liu et al., 2013). Famous herbal teas in pan-China include those are drunk in Yao area (Jin et al., 2018), Lingnan (Liu Y. et al., 2013), Chaoshan (Li et al., 2017), and Fujian (Lin, 2014), and Taiwan (Chang, 2005). Furthermore, this beverage has been closely associated with the prevention and treatment of local common ailments (Li et al., 2017; Tan et al., 2017). The herbal teas in Lingnan (Liu Y. et al., 2013), Chaoshan (Li et al., 2017), and Fujian (Lin, 2014) are called the"cool tea," implying that the herbal teas are used against the hot weather in southern China. The type of herbal tea is also influenced by the traditional culture of various regions, thereby reflecting local characteristics ( $\mathrm{Hu}, 2005)$.

Although the folk plants in Taiwan are widely used in religious rites, bathing, cuisine, and herbal tea, international literature on Taiwanese folk plants is limited. According to previous surveys, there are 1,217 wild or cultivated folk plants with medicinal purposes have been documented in Taiwan (Ministry of Health and Welfare, 2021). The two main herbal teas in Taiwan, bitter tea (kŭ-chá) and green herb tea (qīng-căo-chá), are the two most complicated applications of folk plants in Taiwan. Both of them are usually made by cooking a mixture of medicinal plants (Chang, 2004). The mixtures of medicinal plants, in which there are the mixtures of bioactive compounds, exert synergistic therapeutic effects (Gertsch, 2011; Gras et al., 2018). Herbal teas in Taiwan are believed to have originated in southern China. After arriving in Taiwan, the ingredients of original herbal teas gradually turned into readily available Taiwanese native plants, and then, herbal teas that are suitable for local people were gradually developed (Chen and Lin, 2012).

Although the formulations of bitter tea and green herb tea vary across Taiwanese stores, they are all primarily advertised as having the capacity to "clear heat" (Huang et al., 2020). In the principle of traditional Chinese medicine formulation, one to three medicinal materials play the role of core medicinal materials in an herbal mixture. Other medicinal materials are added to enhance the therapeutic effects, improve the taste, or reduce the side effects of the core plant medicinal materials (Zhou et al., 2016).

According to previous research and surveys, the main components of green herb tea in Taiwan are Platostoma palustre (Blume) A.J.Paton, Bidens pilosa L., Pteris multifida Poir., Mentha arvensis L., Sphagneticola calendulacea (L.) Pruski, and Rhinacanthus nasutus (L.) Kurz (Huang et al., 2020). These tea beverages prepared by mixing and decocting these plants have a refreshing, half bitter/half sweet taste, and are used for quenching thirst and relieving summer heat (Huang et al., 2020). The other Taiwanese main herbal tea, the Taiwanese bitter tea, is also known as liver nourishing tea (yăng-gān-chá), and thick green herb tea (nóng-hóu-qīng-căo-chá). It is characterized by a bitter-dominated taste that is stronger than that of regular green herb tea (Chang, 2005). Although bitter tea has been consumed for hundreds of years in Taiwan, its components and core medicinal materials have not been comprehensively studied, which has impeded the investigation of its efficacy against various health conditions.

Traditional Chinese medicine categorizes medicinal materials according to their property and flavor. The property includes hot, warm, neutral, cool, cold. Hot and warm materials are usually taken to supply energy to the body, while cool and cold materials are taken to drain away the heat from the body (Liu et al., 2020). The flavors are divided into sour, bitter, sweet, spicy, salty, and plain. Flavor is the taste of herbs in the mouth. Each of the flavors has distinct medicinal therapeutic functions. Sour herbs astringe the leakage of fluids and energy; sweet herbs tonify and harmonize; spicy herbs disperse and move; salty herbs soften and purge; plain herbs leech out dampness; and finally, the traditional therapeutic function associated with bitter herbs is heat clearing and removing dampmess (Bensky et al., 2004; Lu et al., 2018; Liu et al.,. 2020). The heat-clearing and dampessdraining effects provided by the bitter taste are used to combat the discomfort caused by the summer rainy season ( $\mathrm{Wu}, 2005)$. Moreover, according to a previous study, the administration of bitter drugs can help treat liver disease (Tsai et al., 2020). For example, Gentiana scabra Bunge, Artemisia capillaris Thunb., and Scutellaria baicalensis Georgi are the most classical and commonly used traditional Chinese medicines for liver disease (Tsai et al., 2020).

Only one of the two main types of herbal tea in Taiwan, the composition and pharmacology of green herb tea constituents have been studied and reported (Huang et al., 2020), whereas those of the raw materials of bitter tea have not yet been elucidated. According to local reference books, most raw materials used in such herbal teas are either cultivated locally or wildly harvested. They require simple process after collection and are decocted after drying (Chang, 2005). However, the formula of Taiwanese bitter tea is not in the public domain, considering that every store keeps their recipe a secret. In the present study, we surveyed sellers of folk herbal medicine in Taiwan. The survey was conducted via interviews using semistructured questionnaires, and information recorded included the current status of the bitter tea market in Taiwan as well as the ingredients and formulations used. The results of the present study can help reveal the ethnopharmacological aspects of bitter tea consumed in Taiwan and facilitate the conservation of such unique cultural resources.

\section{MATERIALS AND METHODS}

\section{Survey Area and Period}

Taiwan, an island in East Asia, is located at $21^{\circ} 45^{\prime}-25^{\circ} 56^{\prime} \mathrm{N}$ and $119^{\circ} 18^{\prime}-124^{\circ} 34^{\prime} \mathrm{E}$. It spans the Tropic of Cancer, covers an area of 


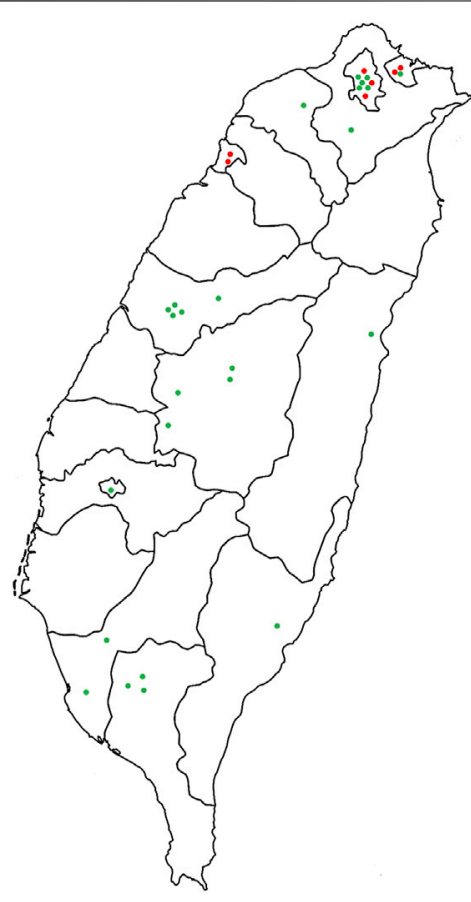

FIGURE 1 | Geographical distribution of 32 stores with available bitter tea formulations. Red dots indicate that the store provided bitter tea formulation but did not receive interviews (seven stores), and green dots indicate that the store provided complete bitter tea formulation and accepted interviews (25 stores).

$36,197 \mathrm{~km}^{2}$, and mostly has a subtropical climate with monsoons, warm and humid summers, and heavy rainfall. In the present study, 59 stores were visited, 25 of which provided information on bitter tea formulations and participated in the interviews. Seven stores only provided information on the bitter tea formula used in their stores but did not give interviews. The 32 sampled stores were distributed all over Taiwan (Figure 1). The survey period was from December 2018 to April 2019, and the entire research activity was reviewed and approved by Taiwan's Central Regional Research Ethics Center (CRREC-107-019) (Supplementary Figure S1).

\section{Interviews and Data Collection}

A semi-structured questionnaire was used for interviews, and the subjects were store owners selling bitter tea. The stores were found online or introduced to us by organizations involved with local medicinal plants. The store owners were invited to participate in the survey, and interviews were conducted on a voluntary basis. With the permission of the informants, written records, audio recordings, and photographs were obtained, and herbal medicines were acquired by the authors. The medicinal materials were then identified using the five senses identification method, with which the expert identify the origin of medicinal materials by touch, taste, smell, hearing, and visual observation, without any equipments
(Hsieh et al., 2017). The specimens of the medicinal materials have been deposited in the herbarium at School of Pharmacy of China Medical University (CMU) in Taichung City, Taiwan.

The information collected by the interviews was divided into two parts. The first part included basic information on the store, including age and sex of the store owner, store location, and how long the store has been in operation. The second part was a semistructured questionnaire consisting of the following questions: 1) what is the composition of bitter tea; 2) what is the traditional therapeutic indication of the bitter tea sold in your store; 3) how was the traditional knowledge of the bitter tea obtained; and 4) does the store sell dry or fresh raw materials needed to make the tea?

\section{Data Collection of Bitter Tea Plant Materials in Taiwan}

All data on the surveyed plant materials were collated, which included the following:

(1) Name, including scientific name, family name, and local name. The scientific and family names of plants are presented according to the Plant List nomenclature (The Royal Botanic Gardens, 2013) and the Plants of the World Online (Royal Botanic Garden, 2021).

(2) Part of the plant used. This was determined by identifying the raw plant materials.

(3) Frequency and use value (UV). Frequency refers to the number of stores using the plant material in the survey, and UV refers to the number of stores using the plant material/total number of stores in the survey as follows (Fu et al., 2018):

$$
\mathrm{UV}=\frac{\sum \mathrm{Ui}}{\mathrm{N}}
$$

Where Ui represents the individual number of the $i$ th medicinal material, and $\mathrm{N}$ represents the total number of bitter tea formulations.

(4) Traditional therapeutic functions. This information was obtained from the Committee on Chinese Medicine and Pharmacy (2003) and the second edition of The Committee on Chinese Medicine and Pharmacy (2011).

(5) The diversity of the medicinal plants was evaluated using Shannon diversity index (Gras et al., 2018), which was calculated as follows:

$$
\text { Shannon diversity index }=-\sum_{i=1}^{n} P i * \log _{2} P i
$$

Where $P i$ represents the proportion of the individual number of the $i$ th medicinal material to the individual number of the total medicinal material. For example, Andrographis paniculata was used in 10 of the formulations, and the total number of individual of all species in this survey is 183. Then the Pi for Andrographis paniculata was obtained by dividing 10 by 183 . Then the 
" $P i * \log _{2} P i "$ for all the medicinal materials in the survey were summed up to get the Shannon diversity index.

(6) Modern pharmacology. Information was obtained by searching for the plant materials in the literature using the PubMed database ${ }^{1}$. Those cited in studies published before April 2021 and at a frequency greater than nine were included.

(7) Comparison between Taiwanese bitter tea and Taiwanese green herb tea. Based on the findings of Huang et al. (2020), we analyzed the similarities and differences between the plant materials used for each tea and further compared differences in flavor, UV, and application in modern pharmacology.

(8) Comparison of herbal tea composition across three regions in southern China. By referring to relevant published articles (Liu et al., 2013; Lin, 2014; Li et al., 2017), a Venn diagram was plotted using an online tool ${ }^{2}$ to analyze the relationships between Taiwanese bitter tea and herbal tea from Lingnan, Chaoshan, and Fujian.

\section{Core Network Analysis of Bitter Tea Use in Taiwan}

Core network analysis was carried out using the Traditional Chinese Medicine Inheritance Support System (TCMISS) v2.5 (Wu et al., 2019; Chao et al., 2020; Wu Z. et al., 2020). Several matching frequency conditions were input into the software to find a suitable network diagram. Establishing connections when combinations of plant materials had a matching frequency greater than four was found the most proper to generate the network that clearly present the core medicinal materials. The length of the connection indicates the degree of matching frequency, and the size of the circles represent the relative UV.

\section{RESULTS}

\section{Respondents' Data and Store Information}

A total of 59 stores selling bitter tea were visited for this study. Among them, 25 participated in interviews and provided the formulations for bitter tea; 7 were unwilling to participate in the interviews but provided bitter tea formulations; and 27 declined to participate in interviews and did not provide formulations. Of the 25 traditional stores that participated in interviews, 50\% have been operating for more than 50 years. The store owners were mainly male (76\%), with ages ranging from 31 to 70 years. Knowledge on bitter tea formulations was mostly passed down through family members (76\%) or apprentices (20\%). All stores sold prepared bitter tea drinks (100\%), 36\% sold dry raw materials, and $48 \%$ sold both dry and fresh raw materials (Supplementary Figure S2). In addition, all bitter tea sellers sold green herb tea.

${ }^{1}$ https://www.ncbi.nlm.nih.gov/pubmed

${ }^{2}$ http://bioinformatics.psb.ugent.be/webtools/Venn/

\section{Plant Materials Used in Bitter Tea and Their Territories in Taiwan}

In the present study, 73 plant materials from 72 plants belonging to 33 families and 67 genera were identified. Among the 73 medicinal materials, $60 \%$ of them were cultivated, $63 \%$ of them can be collected in the wild, and $37 \%$ were both cultivated and wild (Supplementary Table S1). The average number of plants materials in a formulation is 5.7 (Supplementary Table S2). The top five ranking species include Andrographis paniculata (Burm. f.) Nees, Tithonia diversifolia (Hemsl.) A. Gray (both UV = 0.312), Ajuga nipponensis Makino, Ixeris chinensis (Thunb. ex Thunb.) Nakai (both UV $=0.281$ ), and Ilex asprella (Hook. \& Arn.) Champ. ex Benth (UV = 0.25). Regarding the plant families, Asteraceae was the most frequently used, comprising $16.7 \%$ among 72 plants and $75.0 \%$ among 32 formulations, followed by Lamiaceae, comprising $15.3 \%$ among total plants and $65 \%$ among formulations (Figure 2A). The most used plant parts were the whole plant $(41.1 \%)$, followed by the root and rhizome (20.6\%) (Figure 2B).

The Shannon diversity index for all the 73 medicinal materials in Taiwan was 5.73. Among the northern, central, and southern Taiwan, the medicinal materials used in northern Taiwan were the most diverse, with a Shannon diversity index of 5.27. While the Shannon diversity indices of central and southern Taiwan were 4.92 and 4.11, respectively. Moreover, the more the north, the more species of medicinal materials were used. A total of 49 medicinal materials were used in bitter tea in northern Taiwan, while there were only 34 medicinal materials used in Central Taiwan, and even fewer in southern Taiwan, with only 20 medicinal materials (Supplementary Table S3). In these three areas, only nine medicinal materials were commonly used (Figure 2C). They were Tithonia diversifolia (stem), Ixeris chinensis, Rhinacanthus nasutus (L.) Kurz, Orthosiphon aristatus (Blume) Miq., Andrographis paniculata (Burm. f.) Nees, Solanum incanum, Mallotus repandus (Willd.) Muell.-Arg., Sphagneticola calendulacea (L.) Pruski, and Pteris multifida Poir.

\section{Ethnomedicinal Functions of the Bitter Tea}

When asked about the traditional applications of the bitter tea sold at a store, all sellers answered based on traditional concepts in Taiwan folk therapy; the bitter flavor is said to protect the liver, and the main ethnomedicinal function of bitter tea is "clearing heat, protecting the liver, and lowering liver fire." The sellers also stated that bitter tea is a type of folk therapy product for the liver.

The traditional applications of the 73 identified plant materials were revealed, excluding $T$. diversifolia (stem) and A. keiskei since there were no records on their properties and flavors (Supplementary Table S1). The cold, hot, warm, and cool properties were then analyzed for 71 plant materials; cold properties were the most common $(46.5 \%)$ followed by cool (26.8\%), jointly accounting for $73.3 \%$ of the plant material properties (Figure 3A). Moreover, 69\% of the plant materials had a bitter flavor (Figure 3B). According to a comprehensive analysis of the four properties 

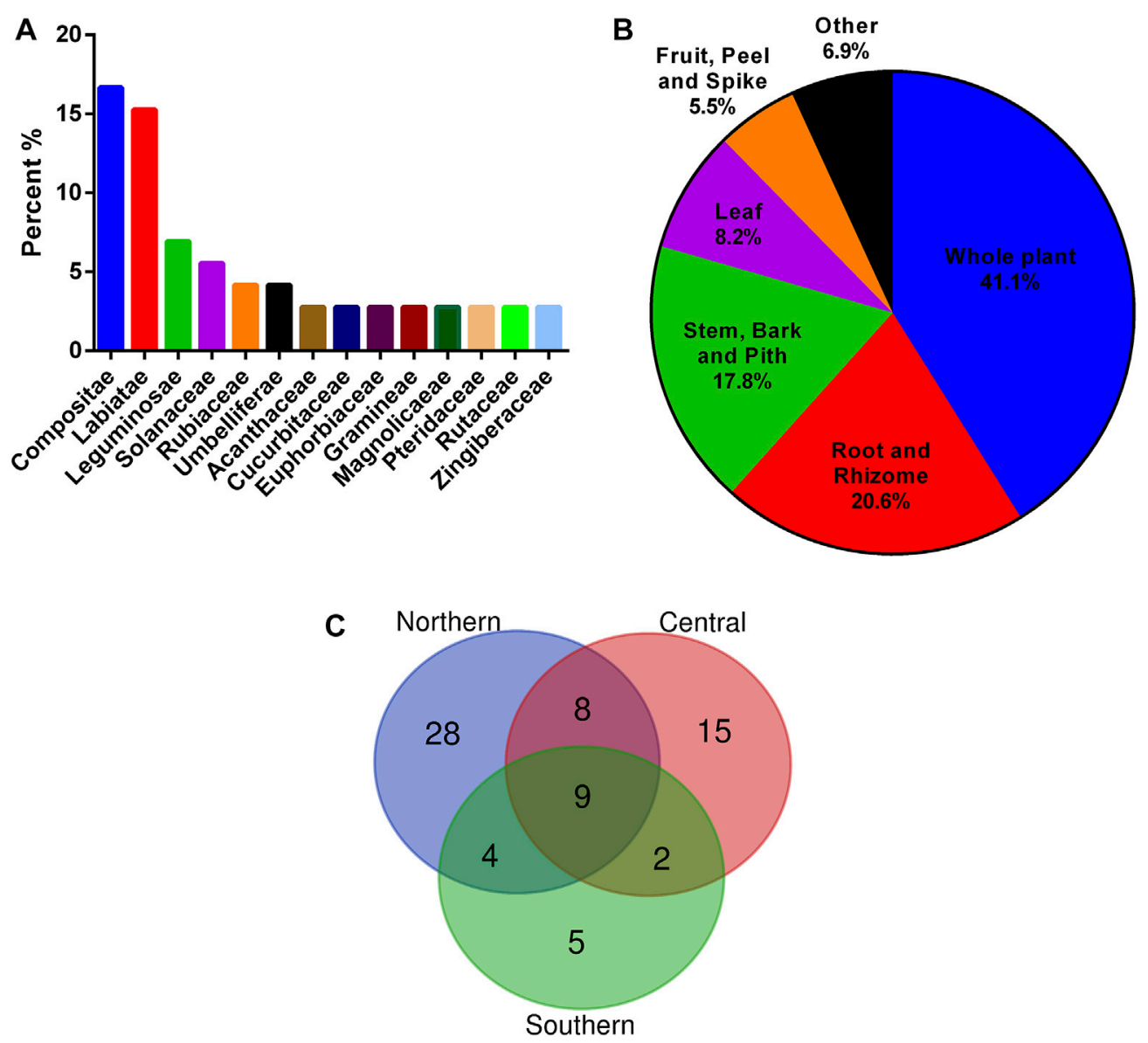

FIGURE 2 | Origin analysis of 73 plant materials used in bitter tea. (A) Families; (B) plant parts; (C) the number of shared plant materials in areas of Taiwan.

and five flavors, cold and bitter plants are the most commonly used for preparing bitter tea in Taiwan (Figure 3C). Analysis of the ethnomedicinal function showed that 63.9, 54.8, 25.0, and $25.0 \%$ of the 73 plant materials had heat-clearing, detoxification, detumescence, and diuresis effects, respectively (Figure 3D).

Among the 24 commonly used plant materials in Taiwanese bitter tea, those with a frequency of use greater than nine were collected to analyze their modern pharmacological applications (Table 1). Anti-inflammation was the most common pharmacological action (Figure 3E), followed by anticancer, antioxidant, antimicrobial, hepatoprotection, and antidiabetic effects.

\section{Comparison Between Taiwanese Bitter Tea and Green Herb Tea}

We next compared the top 15 most commonly used plant materials between Taiwanese bitter tea and green herb tea (Figure 4A) and found an overlap of only four plant materials: Glycyrrhiza uralensis Fisch., $P$. palustre, I. asprella, and Rhinacanthus nasutus (L.) Kurz.
Therefore, the plant materials used vary between these two tea types.

Plant materials with a bitter flavor were then divided into bitter cold and sweet cold; bitter cold plant materials accounted for 37.1 and $21.4 \%$ of the bitter tea and green herb tea plant materials, respectively, whereas sweet cold plants were used in the same proportion of approximately $19 \%$ (Figure 4B). The UV values of the top five most commonly used plant materials were higher for green herb tea than for bitter tea (Figure 4C). Moreover, the composition of green herb tea sold in different stores was shown to have minimal differences and high consistency, whereas the constituents of bitter tea differed among businesses in Taiwan. Therefore, the selection of plant materials for bitter tea is not consistent.

Anti-inflammation is the most published aspect in plant pharmacology research associated with the top 15 most commonly used plant materials in bitter tea and green herb tea (Figure 4D). For green herb tea, this is followed by antioxidant, antimicrobial, and anticancer effects, which is slightly different than that for bitter tea (Figure 4D). 

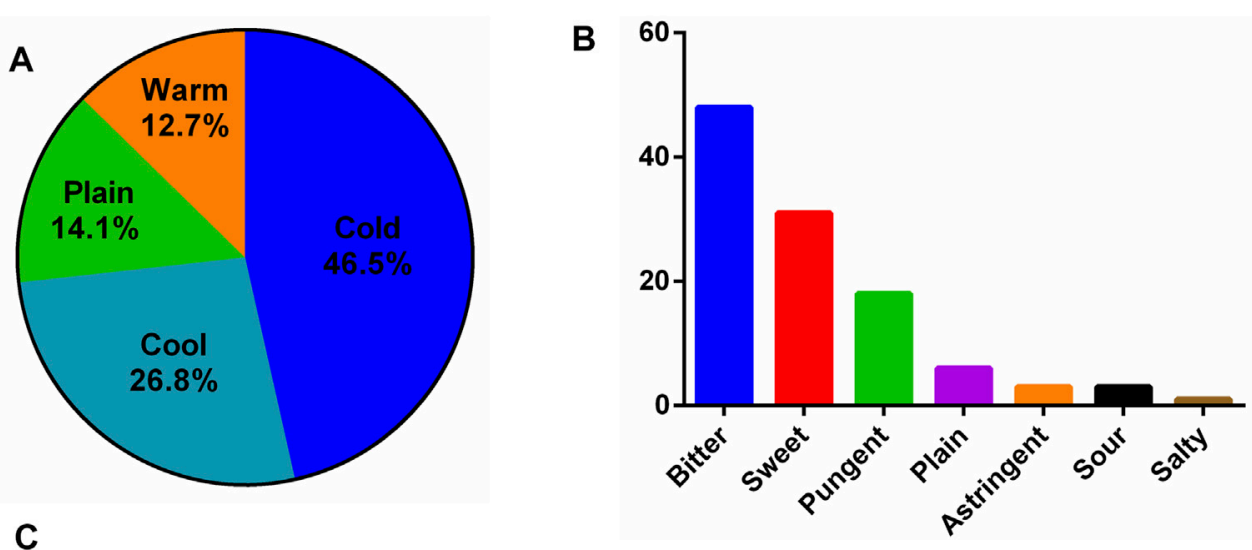

C

\begin{tabular}{|c|c|c|c|c|c|c|c|}
\hline & Sour & Bitter & Sweet & $\begin{array}{c}\text { Pungen } \\
\mathrm{t}\end{array}$ & Salty & Plain & $\begin{array}{c}\text { Astring } \\
\text { ent }\end{array}$ \\
\hline Cold & 1.43 & 37.14 & 18.57 & 7.14 & 0.00 & 4.29 & 1.43 \\
\hline Cool & 0.00 & 18.57 & 14.29 & 7.14 & 1.43 & 2.86 & 0.00 \\
\hline Neutral & 0.00 & 7.14 & 8.57 & 4.29 & 0.00 & 1.43 & 1.43 \\
\hline Warm & 2.86 & 5.71 & 2.86 & 7.14 & 0.00 & 0.00 & 1.43 \\
\hline Hot & 0.00 & 0.00 & 0.00 & 0.00 & 0.00 & 0.00 & 0.00 \\
\hline
\end{tabular}
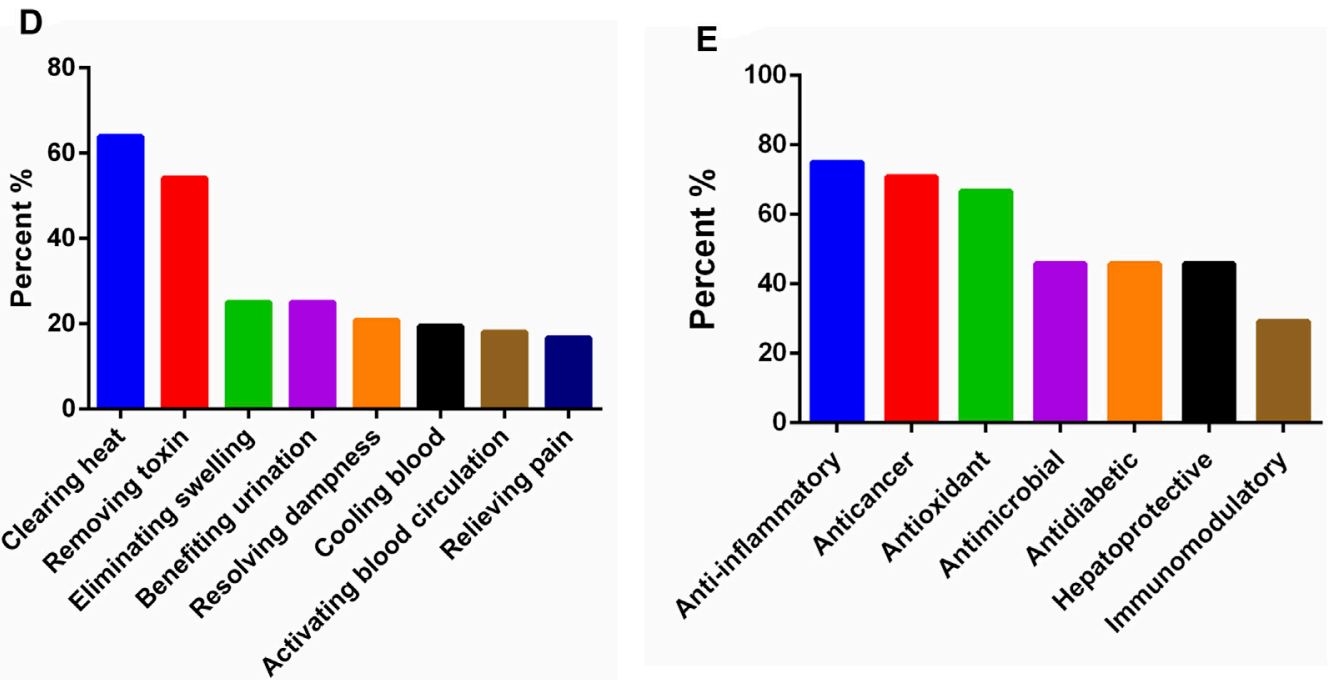

FIGURE 3 | Traditional and modern pharmacological analysis of plant materials used in Taiwanese bitter tea. (A) Nature; (B) flavor; (C) comprehensive analysis of nature and flavor (\%); (D) ethnomedicinal effects; (E) modern pharmacological applications of plant materials with a frequency greater than nine.

\section{Comparison of Taiwanese Bitter Tea With That of Herbal Tea From Lingnan, Chaoshan, and Fujian}

In the south of China near Taiwan, there are other local herbal teas (liang-cha; cool tea) that are commonly consumed. Comparisons of Taiwanese bitter tea with herbal teas from the Lingnan area (Liu Y. et al., 2013), Chaoshan area (Li et al., 2017), and Fujian (Lin, 2014) revealed that the plant materials used in herbal teas vary across the four locations.
Notably, $35(48.0 \%)$ of the plant materials used in bitter tea, according to the survey, were limited to Taiwan (Figure 5).

\section{Analysis of High-Frequency Drug Pairs and Core Network Analysis of Taiwanese Bitter Tea}

To identify the drug pairs used in high frequencies, we performed core network analysis. Eight drug pairs appeared more than four times in 32 formulations and included nine medicinal plant materials 
TABLE 1 | Modern pharmacological effects and traditional use of highly cited plant materials used in Taiwanese bitter tea (UV > 0.09).

\begin{tabular}{|c|c|c|c|c|c|c|}
\hline $\begin{array}{l}\text { Voucher } \\
\text { specimen } \\
\text { numberCMUBT- } \\
\text { Xa }\end{array}$ & Scientific name & Family & $\begin{array}{l}\text { Local } \\
\text { name }\end{array}$ & $\begin{array}{l}\text { Use } \\
\text { value } \\
(\%)\end{array}$ & $\begin{array}{l}\text { Related research } \\
\text { on PubMed }\end{array}$ & Traditional use \\
\hline 1 & $\begin{array}{l}\text { Andrographis paniculata (Burm. } \\
\text { f.) Nees }\end{array}$ & Acanthaceae & $\begin{array}{l}\text { Chuān Xīn } \\
\text { lián (穿 } \\
\text { 心蓮) }\end{array}$ & 0.31 & $\begin{array}{l}\text { Antioxidant Mussard et al. (2020), } \\
\text { anti-inflammatory Burgos et al. } \\
\text { (2020), anti-hyperglycemic } \\
\text { Wediasari et al. (2020), } \\
\text { hepatoprotective Toppo et al. } \\
\text { (2021), antimicrobial Hossain et al. } \\
\text { (2021), anticancer Malik et al. } \\
\text { (2021), immunomodulatory Liu et al. } \\
\text { (2020), cardioprotective Lin et al. } \\
\text { (2020), anti-hyperlipidemic, sexual } \\
\text { functions and contraceptive } \\
\text { Hossain et al. (2014), antiviral } \\
\text { Jadhav and Karuppayil, (2021), } \\
\text { Sa-Ngiamsuntorn et al. (2021), anti- } \\
\text { snake venom Nayak et al. (2021), } \\
\text { antimalarial Hassan et al. (2019), } \\
\text { anti-Alzheimer Lu et al. (2019), and } \\
\text { anti-obesity Chen et al. (2020) }\end{array}$ & $\begin{array}{l}\text { Clears heat, resolves toxins, } \\
\text { disperses swelling, and } \\
\text { relieves pain }\end{array}$ \\
\hline 2 & $\begin{array}{l}\text { Tithonia diversifolia (Hemsl.) A. } \\
\text { Gray (stem) }\end{array}$ & $\begin{array}{l}\text { Asteraceae } \\
\text { (Compositae) }\end{array}$ & $\begin{array}{l}\text { Wǔ zhǎo jin } \\
\text { yīng (五爪 } \\
\text { 金英) }\end{array}$ & 0.31 & $\begin{array}{l}\text { Anti-inflammatory Broering et al. } \\
\text { (2019), antimicrobial, antioxidant } \\
\text { Ferreira Farias et al. (2019), } \\
\text { anticancer Lu et al. (2017), analgesic } \\
\text { Owoyele et al. (2004), anti- } \\
\text { hyperlipidemic, antidiabetic Miura } \\
\text { et al. (2005), antiviral Maregesi et al. } \\
\text { (2010), gastroprotective } \\
\text { Sanchez-Mendoza et al. (2011), } \\
\text { immunomodulatory Ejelonu et al. } \\
\text { (2017), antimalarial Afolayan et al. } \\
\text { (2016), hepatoprotective Nguepi } \\
\text { et al. (2021), anti-obesity, } \\
\text { antiemetic, anti-diarrheal, anti- } \\
\text { leishmania, and anti-trypanosoma } \\
\text { Mabou Tagne et al. (2018) }\end{array}$ & $-b$ \\
\hline 3 & Ajuga nipponensis Makino & Lamiaceae & $\begin{array}{l}\text { Bái mǎ wú } \\
\text { gōng (白馬 } \\
\text { 蜗蚣) }\end{array}$ & 0.28 & $\begin{array}{l}\text { Antioxidant, hepatoprotective Hsieh } \\
\text { et al. (2016), and anti- } \\
\text { osteoclastogenic Wang, H. et al. } \\
\text { (2021) }\end{array}$ & $\begin{array}{l}\text { Disperses inflammation, cools } \\
\text { the blood, and joins bone } \\
\text { (Jiēgǔ) }\end{array}$ \\
\hline 4 & Ixeris chinensis (Thunb.) Nakai & $\begin{array}{l}\text { Asteraceae } \\
\text { (Compositae) }\end{array}$ & $\begin{array}{l}\text { Xiăo jīn yīng } \\
\text { (小金英) }\end{array}$ & 0.28 & $\begin{array}{l}\text { Anticancer Xu et al. (2016), antiviral, } \\
\text { hepatoprotective Shih et al. (2014), } \\
\text { immunomodulatory Li et al. (2020), } \\
\text { and antileukemic Chiang et al. } \\
\text { (2004) }\end{array}$ & $\begin{array}{l}\text { Clears heat, promotes } \\
\text { urination, and calms the spirit }\end{array}$ \\
\hline 5 & $\begin{array}{l}\text { Ilex asprella (Hook. \& Arn.) } \\
\text { Champ. ex Benth }\end{array}$ & Aquifoliaceae & $\begin{array}{l}\text { Wàn diăn } \\
\text { jin (萬點金) }\end{array}$ & 0.25 & $\begin{array}{l}\text { Anti-inflammatory Yang et al. (2018), } \\
\text { anticancer Li et al. (2018), } \\
\text { immunoregulatory Meng et al. } \\
\text { (2018), lung-protective Dai et al. } \\
\text { (2014), anti-hyperlipidemic Hu et al. } \\
\text { (2012), and antiviral Zhang et al. } \\
\text { (2018) }\end{array}$ & $\begin{array}{l}\text { Clears heat, promotes } \\
\text { urination, and calms the spirit }\end{array}$ \\
\hline
\end{tabular}

(Continued on following page) 
TABLE 1 | (Continued) Modern pharmacological effects and traditional use of highly cited plant materials used in Taiwanese bitter tea (UV > 0.09).

\begin{tabular}{|c|c|c|c|c|c|c|}
\hline $\begin{array}{l}\text { Voucher } \\
\text { specimen } \\
\text { numberCMUBT- } \\
\text { Xa }\end{array}$ & Scientific name & Family & $\begin{array}{l}\text { Local } \\
\text { name }\end{array}$ & $\begin{array}{l}\text { Use } \\
\text { value } \\
(\%)\end{array}$ & $\begin{array}{c}\text { Related research } \\
\text { on PubMed }\end{array}$ & Traditional use \\
\hline 6 & $\begin{array}{l}\text { Bombax ceiba L.[Bombax } \\
\text { malabaricum DC.] }\end{array}$ & $\begin{array}{l}\text { Malvaceae } \\
\text { [Bombacaceae] }\end{array}$ & $\begin{array}{l}\text { Mù mián } \\
\text { gēn (木 } \\
\text { 棉根) }\end{array}$ & 0.22 & $\begin{array}{l}\text { Antidiabetic Bhargava and Shah } \\
\text { (2020), antioxidant Komati et al. } \\
\text { (2020), anti-inflammatory, } \\
\text { antisteatotic Arafa et al. (2019), } \\
\text { promotes osteoblast proliferation } \\
\text { Chauhan et al. (2018), antimicrobial } \\
\text { Shah et al. (2018), hepatoprotective } \\
\text { Lee et al. (2017), anticancer Tundis } \\
\text { et al. (2014), anti-obesity Gupta } \\
\text { et al. (2013), promotes male sexual } \\
\text { function, and antiviral Wang et al. } \\
\text { (2013) }\end{array}$ & $\begin{array}{l}\text { Dispels wind, stops itchiness, } \\
\text { dispels wind-dampness, } \\
\text { clears heat, resolves toxins, } \\
\text { removes stasis, and relives } \\
\text { pain }\end{array}$ \\
\hline 7 & Solanum incanum L & Solanaceae & $\begin{array}{l}\text { Huáng shuĩ } \\
\text { qié(黃水茄) }\end{array}$ & 0.19 & $\begin{array}{l}\text { Anticancer Wu, Y.H. et al. (2015), Yu } \\
\text { et al. (2017), Al-Emam et al. (2018) } \\
\text { and antimicrobial Lashin et al. (2021) }\end{array}$ & $\begin{array}{l}\text { Disperses inflammation, } \\
\text { resolves toxins, dispels wind, } \\
\text { relieves pain, clears heat, and } \\
\text { disperses inflammation }\end{array}$ \\
\hline 8 & $\begin{array}{l}\text { Mallotus repandus (Willd.) } \\
\text { Muell.-Arg }\end{array}$ & Euphorbiaceae & $\begin{array}{l}\text { Tǒng jiāo } \\
\text { téng (桶 } \\
\text { 交藤) }\end{array}$ & 0.16 & $\begin{array}{l}\text { Analgesic Hasan et al. (2014), anti- } \\
\text { inflammatory Hasan et al. (2014), } \\
\text { antioxidant Lin et al. (1995), and } \\
\text { hepatoprotective Mondal et al. } \\
\text { (2020) }\end{array}$ & $\begin{array}{l}\text { Clears heat, resolves the } \\
\text { exterior, benefits the throat, } \\
\text { prevents rashes, improves } \\
\text { digestion, disperses swelling, } \\
\text { and stops itch }\end{array}$ \\
\hline 9 & Physalis angulata L & Solanaceae & $\begin{array}{l}\text { Dēng long } \\
\text { căo (燈 } \\
\text { 籠草) }\end{array}$ & 0.16 & $\begin{array}{l}\text { Immunomodulatory Vieceli et al. } \\
\text { (2021), anti-inflammatory Sun, C.P. } \\
\text { et al. (2017a), Sun, C.P. et al. } \\
\text { (2017b), Wang, L. et al. (2021), } \\
\text { antiproliferative Sun, C.P. et al. } \\
\text { (2017b), Chairissy et al. (2019), } \\
\text { antioxidant Adewoye et al. (2016), } \\
\text { reno-restorative Adewoye et al. } \\
\text { (2016), antiparasitic Meira et al. } \\
\text { (2015), antileishmanial Da Silva et al. } \\
\text { (2018), and anticancer Ma et al. } \\
\text { (2017) }\end{array}$ & $\begin{array}{l}\text { Clears heat, resolves toxins, } \\
\text { disperses swelling, and } \\
\text { removes stasis }\end{array}$ \\
\hline
\end{tabular}

\section{Bái hè líng} zhī(白鶴

靈芝)
Anticancer Siripong et al. (2006), Kupradinun et al. (2009), Siripong et al. (2009), Horii et al. (2012), Siripong et al. (2012), Boueroy et al. (2018), anti-obesity Ngoc et al. (2019), anti-glycation Shah et al. (2017), neuroprotective Chuang et al. (2017), acetylcholinesterase inhibitor Brimson and Tencomnao, (2011), Brimson et al. (2012), Chang, C.Z. et al. (2016), Boonyaketgoson et al. (2018), antioxidant Brimson et al. (2012), Shah et al. (2017), Zhao et al. (2019), antidiabetic Shah et al. (2017), Visweswara Rao et al. (2013a), neuraminidase inhibitor Kwak et al. (2018), hepatoprotective Visweswara Rao et al. (2013b), antiinflammatory Zhao et al. (2019), antimicrobial Kernan et al. (1997), Puttarak et al. (2010), Ngoc et al. (2019), anti-allergic Tewtrakul et al. (2009), immunomodulatory

Punturee et al. (2005), anti-
Moistens the lung and stops coughing, calms the liver and reduces fire, disperses swelling, resolves toxins, kills worms, and stops itchiness 
TABLE 1 | (Continued) Modern pharmacological effects and traditional use of highly cited plant materials used in Taiwanese bitter tea (UV > 0.09).

\begin{tabular}{|c|c|c|c|c|c|c|}
\hline $\begin{array}{l}\text { Voucher } \\
\text { specimen } \\
\text { numberCMUBT- }\end{array}$ & Scientific name & Family & $\begin{array}{l}\text { Local } \\
\text { name }\end{array}$ & $\begin{array}{c}\text { Use } \\
\text { value } \\
(\%)\end{array}$ & $\begin{array}{c}\text { Related research } \\
\text { on PubMed }\end{array}$ & Traditional use \\
\hline
\end{tabular}

Parkinson's Saleem et al. (2021), and antifungal Jeenkeawpieam et al. (2020)

\begin{tabular}{|c|c|c|c|c|c|}
\hline 11 & Sigesbeckia orientalis L & $\begin{array}{l}\text { Asteraceae } \\
\text { (Compositae) }\end{array}$ & $\begin{array}{l}\text { Kǔ căo (苦 } \\
\text { 草)Xi liàn } \\
\text { căo (狶 } \\
\text { 薟草) }\end{array}$ & 0.16 & $\begin{array}{l}\text { Antimicrobial, anti-allergic, } \\
\text { antithrombotic Wang, Q. et al. } \\
\text { (2021), anti-inflammatory Nguyen } \\
\text { et al. (2017), Chu et al. (2018), } \\
\text { Engels et al. (2020), } \\
\text { antihyperuricemic Nguyen et al. } \\
\text { (2017), analgesic Nguyen et al. } \\
\text { (2017), and anticancer Sun and } \\
\text { Wang, (2006), Chang, C.C. et al. } \\
\text { (2016) }\end{array}$ \\
\hline
\end{tabular}

\begin{tabular}{|c|c|c|c|c|c|}
\hline 12 & $\begin{array}{l}\text { Boehmeria nivea (L.) } \\
\text { Gaudich.[Boehmeria nivea (L.) } \\
\text { Gaudich. var. tenacissima } \\
\text { (Gaudich.) Miq.] }\end{array}$ & Urticaceae & $\begin{array}{l}\text { Shān zhù } \\
\text { má(山苧麻) }\end{array}$ & 0.13 & $\begin{array}{l}\text { Anti-inflammatory Lim et al. (2020), } \\
\text { laxative, antioxidant Lee et al. } \\
\text { (2020), and antiproliferative Wang } \\
\text { et al. (2019) }\end{array}$ \\
\hline 13 & Glycyrrhiza uralensis Fisch & Leguminosae & $\begin{array}{l}\text { Gān căo } \\
\text { (甘草) }\end{array}$ & 0.13 & $\begin{array}{l}\text { Anticancer, antiulcer, spasmolytic, } \\
\text { hepatoprotective, anti- } \\
\text { inflammatory, antimicrobial Jiang } \\
\text { et al. (2020), and anti-allergic Fouladi } \\
\text { et al. (2019) }\end{array}$ \\
\hline
\end{tabular}

\begin{tabular}{|c|c|c|c|c|c|}
\hline 14 & $\begin{array}{l}\text { Orthosiphon aristatus } \\
\text { (Blume) Miq }\end{array}$ & Lamiaceae & $\begin{array}{l}\text { Huà shí } \\
\text { căo (化 } \\
\text { 石草) }\end{array}$ & 0.13 & $\begin{array}{l}\text { Genoprotective Al-Dualimi et al. } \\
\text { (2018), antibacterial Al-Dualimi et al. } \\
\text { (2018), antidiabetic Damsud et al. } \\
\text { (2014), antioxidant Hsu et al. (2010), } \\
\text { anti-inflammatory Hsu et al. (2010), } \\
\text { and antihypertensive Matsubara } \\
\text { et al. (1999), Ohashi et al. (2000) }\end{array}$ \\
\hline
\end{tabular}

\begin{tabular}{|c|c|c|c|c|c|c|}
\hline 15 & $\begin{array}{l}\text { Platostoma palustre (Blume) } \\
\text { A.J.Paton [Mesona chinensis } \\
\text { Benth.] }\end{array}$ & Lamiaceae & $\begin{array}{l}\text { Xiān căo } \\
\text { (仙草) }\end{array}$ & 0.13 & $\begin{array}{l}\text { Antidiabetic Adisakwattana et al. } \\
\text { (2014), Chusak et al. (2014), Liu S. } \\
\text { et al. (2018), Yuris et al. (2019) and } \\
\text { antioxidant Huang et al. (2019), } \\
\text { Huang, L. et al. (2020) }\end{array}$ & $\begin{array}{l}\text { Clears heat and resolves } \\
\text { toxins }\end{array}$ \\
\hline 16 & $\begin{array}{l}\text { Bidens pilosa L.[Bidens pilosa L. } \\
\text { var. radiata Sch. Bip.] }\end{array}$ & $\begin{array}{l}\text { Asteraceae } \\
\text { (Compositae) }\end{array}$ & $\begin{array}{l}\text { Xián fēng } \\
\text { cǎo (咸 } \\
\text { 豐草) }\end{array}$ & 0.09 & $\begin{array}{l}\text { Anticancer Arantes et al. (2021), } \\
\text { gastroprotective Alvarez et al. } \\
\text { (1999), Horiuchi et al. (2010), Anti- } \\
\text { diabetic Chien et al. (2009), Ubillas } \\
\text { et al. (2000) Anti-allergy Matsumoto } \\
\text { et al. (2009), anti-inflammatory Hong } \\
\text { et al. (2021), Xin et al. (2021) Anti- } \\
\text { malaria Nadia et al. (2020) Anti- } \\
\text { microbial Chiavari-Frederico et al. } \\
\text { (2020) anti-coccidial, Yang et al. } \\
\text { (2019), hepatoprotective Pegoraro } \\
\text { et al. (2021), and anti-hypertensive } \\
\text { Bilanda et al. (2017) }\end{array}$ & $\begin{array}{l}\text { Clears heat, resolves toxins, } \\
\text { promotes urination, and } \\
\text { reduces jaundice }\end{array}$ \\
\hline 17 & Elephantopus scaber L & $\begin{array}{l}\text { Asteraceae } \\
\text { (Compositae) }\end{array}$ & $\begin{array}{l}\text { Ding shù } \\
\text { wū(丁豎桍) }\end{array}$ & 0.09 & $\begin{array}{l}\text { Anticancer Bai et al. (2020), Pandey } \\
\text { et al. (2020), hepatoprotective } \\
\text { Sulistyani and Nurkhasanah, (2020), } \\
\text { anti-inflammatory Fu et al. (2020), Qi } \\
\text { et al. (2020), antioxidant Aslam et al. } \\
\text { (2016), antidiabetic, antimicrobial, }\end{array}$ & $\begin{array}{l}\text { Clears heat, resolves toxins, } \\
\text { promotes urination, and } \\
\text { disperses swelling }\end{array}$ \\
\hline
\end{tabular}


TABLE 1 | (Continued) Modern pharmacological effects and traditional use of highly cited plant materials used in Taiwanese bitter tea (UV > 0.09).

\begin{tabular}{|c|c|c|c|c|c|c|}
\hline $\begin{array}{l}\text { Voucher } \\
\text { specimen } \\
\text { numberCMUBT- } \\
\text { Xa }\end{array}$ & Scientific name & Family & $\begin{array}{l}\text { Local } \\
\text { name }\end{array}$ & $\begin{array}{c}\text { Use } \\
\text { value } \\
(\%)\end{array}$ & $\begin{array}{l}\text { Related research } \\
\text { on PubMed }\end{array}$ & Traditional use \\
\hline
\end{tabular}

\begin{tabular}{|c|c|c|c|c|c|}
\hline & & & & & $\begin{array}{l}\text { and analgesic Hiradeve and } \\
\text { Rangari, (2014) }\end{array}$ \\
\hline 18 & $\begin{array}{l}\text { Momordica charantia } \\
\text { L.[Momordica charantia L. var. } \\
\text { abbreviata Ser.] }\end{array}$ & Cucurbitaceae & $\begin{array}{l}\text { Shān kǔ } \\
\text { guā(山 } \\
\text { 苦瓜) }\end{array}$ & 0.09 & $\begin{array}{l}\text { Anti-obesity Fan et al. (2021), anti- } \\
\text { fatigue Hsiao et al. (2017), anti- } \\
\text { inflammatory Perera et al. (2021), } \\
\text { Tsai et al. (2016), antioxidant Akyuz } \\
\text { et al. (2020), cell-protective Tsai } \\
\text { et al. (2014), antimelanogenic Tsai } \\
\text { et al. (2014), antidiabetic Kulkarni } \\
\text { et al. (2021), and anticancer Ehigie } \\
\text { et al. (2021) }\end{array}$ \\
\hline
\end{tabular}

\begin{tabular}{|c|c|c|c|c|c|c|}
\hline 19 & Mucuna macrocarpa Wall & Leguminosae & $\begin{array}{l}\text { Xiě téng } \\
\text { (血藤) }\end{array}$ & 0.09 & Antileukemic Lu et al. (2010) & $\begin{array}{l}\text { Cures rheumatic pain } \\
\text { (backache) }\end{array}$ \\
\hline 20 & $\begin{array}{l}\text { Oldenlandia diffusa (Willd.) } \\
\text { Roxb.[Hedyotis diffusa Willd.] }\end{array}$ & Rubiaceae & $\begin{array}{l}\text { Bái huā shé } \\
\text { shé căo (白 } \\
\text { 花蛇舌草) }\end{array}$ & 0.09 & $\begin{array}{l}\text { Anticancer Chung et al. (2017), } \\
\text { immunomodulatory, antioxidant } \\
\text { Chen et al. (2016), and anti- } \\
\text { inflammatory Zhu et al. (2018) }\end{array}$ & $\begin{array}{l}\text { Clears heat, resolves toxins, } \\
\text { engenders fluid, relieves thirst, } \\
\text { and invigorates the blood }\end{array}$ \\
\hline 21 & Pteris multifida Poir & Pteridaceae & $\begin{array}{l}\text { Fèng wěi } \\
\text { căo (鳳 } \\
\text { 尾草) }\end{array}$ & 0.09 & $\begin{array}{l}\text { Anti-cancer Kim et al. (2017), anti- } \\
\text { neuroinflammatory Kim et al. (2016), } \\
\text { anti-hyperlipidemic Wang et al. } \\
\text { (2010), free radical-scavenging } \\
\text { activity Wang et al. (2007), and anti- } \\
\text { inflammatory Yin et al. (2018) }\end{array}$ & $\begin{array}{l}\text { Clears heat, promotes } \\
\text { urination, cools the blood, and } \\
\text { resolves toxins }\end{array}$ \\
\hline
\end{tabular}

$22 \quad$ Scutellaria barbata D. Don Lamiaceae Bàn zhī lián $\quad 0.09 \quad$ Anti-inflammatoryAkyuz et al. (半枝蓮) (2020), Liu H.L. et al. (2018), anticancerChen et al. (2017), Gao et al. (2014), Jin et al. (2017), Kan et al. (2017), Lin et al. (2017), Marconett et al. (2010), Ozmen et al. (2010), Sun, P. et al. (2017), Zhang et al. (2017), Zhang et al. (2021), antiproliferative Kim et al. (2008), Wu and Chen, (2009), attenuates diabetic retinopathy Mei et al. (2017), neuroprotective Wu et al. (2016), improves cognition Zhang and Li, (2016), antimicrobial Sato et al. (2000), Wu, T. et al. (2015), Yu et al. (2004), acaricidal Yang et al. (2013), and antioxidant Ye and Huang, (2012)

\begin{tabular}{|c|c|c|c|c|c|c|}
\hline 23 & $\begin{array}{l}\text { Sphagneticola calendulacea (L.) } \\
\text { Pruski [Wedelia chinensis } \\
\text { (Osbeck) Merr.] }\end{array}$ & $\begin{array}{l}\text { Asteraceae } \\
\text { (Compositae) }\end{array}$ & $\begin{array}{l}\text { Huáng huā } \\
\text { mì cài (黃花 } \\
\text { 蜜菜) }\end{array}$ & 0.09 & $\begin{array}{l}\text { Antidiabetic Chen et al. (2021), Thao } \\
\text { et al. (2018), anticancer Huang et al. } \\
\text { (2016), Lin et al. (2007), Liu, M. et al. } \\
\text { (2013), Tsai et al. (2009), Tsai et al. } \\
\text { (2015), Tsai et al. (2017a), Tsai et al. } \\
\text { (2017b), neuroprotective Lin et al. } \\
\text { (2014), antibacterial Darah et al. } \\
\text { (2013), anti-inflammatory Darah } \\
\text { et al. (2013), and antioxidant } \\
\text { Manjamalai and Berlin Grace, (2012) }\end{array}$ & $\begin{array}{l}\text { Clears heat, resolves toxins, } \\
\text { eliminates stasis, and } \\
\text { disperses swelling }\end{array}$ \\
\hline 24 & $\begin{array}{l}\text { Tithonia diversifolia (Hemsl.) } \\
\text { A.Gray (leaf) }\end{array}$ & $\begin{array}{l}\text { Asteraceae } \\
\text { (Compositae) }\end{array}$ & $\begin{array}{l}\text { Wǔ zhǎo jin } \\
\text { yīng yè(五 } \\
\text { 爪金英葉) }\end{array}$ & 0.09 & $\begin{array}{l}\text { Hepatoprotective Nguepi et al. } \\
\text { (2021), cardioprotective Ide et al. } \\
\text { (2020), antioxidant, antimicrobial } \\
\text { Ferreira Farias et al. (2019), } \\
\text { antidiabetic, immunomodulatory, } \\
\text { analgesic, antimalarial, anti-obesity, }\end{array}$ & $\begin{array}{l}\text { Clears heat, resolves toxins, } \\
\text { disperses swelling, and } \\
\text { relieves pain }\end{array}$ \\
\hline
\end{tabular}


TABLE 1 | (Continued) Modern pharmacological effects and traditional use of highly cited plant materials used in Taiwanese bitter tea (UV > 0.09).

\begin{tabular}{|c|c|c|c|c|c|c|}
\hline $\begin{array}{l}\text { Voucher } \\
\text { specimen } \\
\text { numberCMUBT- }\end{array}$ & Scientific name & Family & $\begin{array}{l}\text { Local } \\
\text { name }\end{array}$ & $\begin{array}{l}\text { Use } \\
\text { value } \\
(\%)\end{array}$ & $\begin{array}{c}\text { Related research } \\
\text { on PubMed }\end{array}$ & Traditional use \\
\hline
\end{tabular}

Xa

anti-hyperlipidemic,

gastroprotective, antiemetic,

antidiarrheal, antileishmanial, anti-

trypanosomal, antivenin, and

antiviral Mabou Tagne et al. (2018)

${ }^{a}$ CMUBT, china medical university bitter tea.

${ }^{b}$ No records for the stem of T. diversifolia in the Committee on Chinese Medicine and Pharmacy (2003) and the second edition of The Committee on Chinese Medicine and Pharmacy (2011).

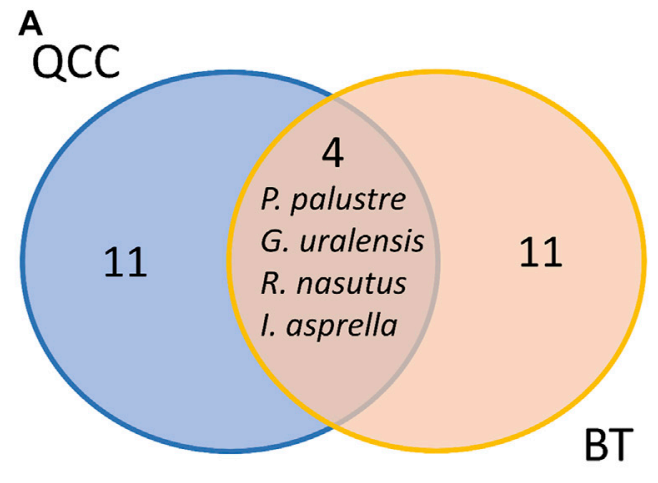

A

C

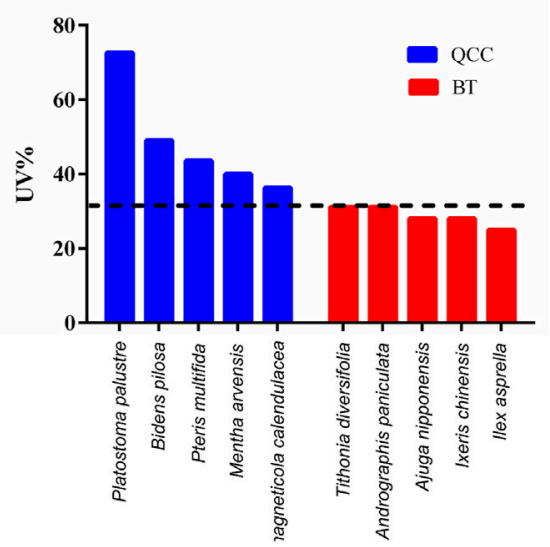

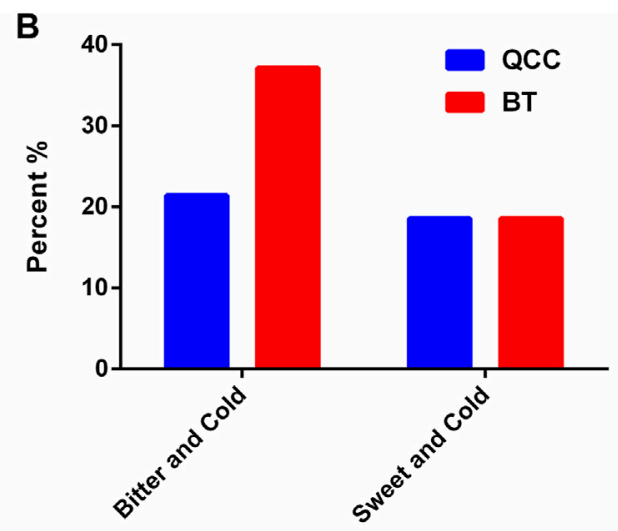

D

\begin{tabular}{|c|c|c|}
\hline & QCC & BT \\
\hline 1 & Anti-inflammatory & Anti-inflammatory \\
\hline & Anti-oxidant & Anti-oxidant \\
\hline & Anti-microbial & Anti-cancer \\
\hline & Anti-cancer & Hepatoprotective \\
\hline & Anti-diabetic & Anti-microbial \\
\hline 6 & Hepatoprotective & Anti-diabetic \\
\hline
\end{tabular}

FIGURE 4 | Comparisons between Taiwanese bitter tea and green herb tea. (A) Venn diagram of the top 15 most commonly used plant materials in bitter tea and green herb tea. (B) Comparison of the nature and flavor of the plant materials. (C) Use values of the top five most commonly used plant materials (1-5 indicate the top five commonly used plant materials). The horizontal dotted line indicates the highest UV of BT. (D) Modern pharmacological applications of the top 15 most commonly used plant materials. QCC, qing-cao-cha (green herb tea); BT, bitter tea.

from A. paniculata, I. asprella, T. diversifolia, A. nipponensis, Mallotus repandus (Willd.) Muell.-Arg., Bombax ceiba L., Solanum incanum L., and $I$. chinensis. The most commonly used drug pairs were $A$. paniculata-A. nipponensis and A. paniculata-I. asprella, with a frequency of occurrence of 5 in 32 formulations.
We constructed a network diagram (Figure 6) of the core components of Taiwanese bitter tea using TCMISS v2.5, and found that the core medicinal materials were from $A$. nipponensis and $T$. diversifolia. The medicinal plants often matched with $A$. nipponensis were $I$. chinensis, A. paniculata, and T. diversifolia, 


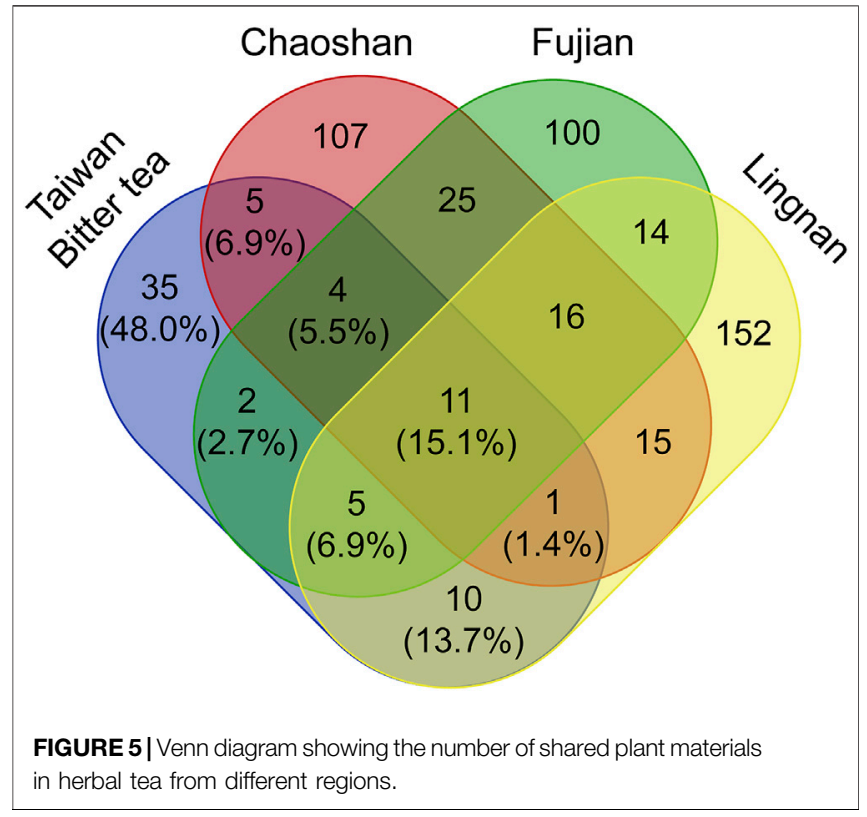

whereas those often matched with $T$. diversifolia were $A$. nipponensis, I. asprella, and $M$. repandus surrounded by $T$. diversifolia, $M$. repandus, and S. incanum.

\section{DISCUSSION}

In ethnopharmacological research, the method of field surveys have been used to explore the medicinal plants and traditional medicines prescribed for certain diseases in different regions. Topics related to medicinal botanicals that employed field investigation in the past include quality control of authentic medicinal materials (Zhao et al., 2012), green herb tea (Huang et al., 2020), herbal tea (Liu et al., 2013; Li et al., 2017), medicinal diets (Tan et al., 2017), and herbal compositions of lactation promoting herbs (Chao et al., 2020). In the present study, the field survey was adopted on Taiwanese bitter tea. We surveyed and analyzed the formulations of Taiwanese bitter tea, a special herbal tea commonly consumed in Taiwan, and revealed its current status in terms of ingredients and function.

Most of the identified plant materials in the study belonged to the family Asteraceae and Lamiaceae. The high involvement of Asteraceae and Lamiaceae in Taiwanese bitter tea is consistent with some other investigations in folk medicine outside Taiwan. They are two of the "hot families" in the medical plants (Saslis-Lagoudakis et al., 2011). Medicinal plants of the two families are dominant in Mediterranean (Gras et al., 2018), Ugandan (Tugume and Nyakoojo, 2019), Turkish (Güneş et al., 2017), Lebanese (Baydoun et al., 2015), Peruvian (Rehecho et al., 2011), Jordanian (Alzweiri et al., 2011), and Algerian (Rachid et al., 2012) folk medicines. Asteraceae exhibits high environmental adaptability and hosts a large number of naturalized and invasive plants globally. In addition, the climate in Taiwan is conducive for Asteraceae growth, and plants in this family are the third most

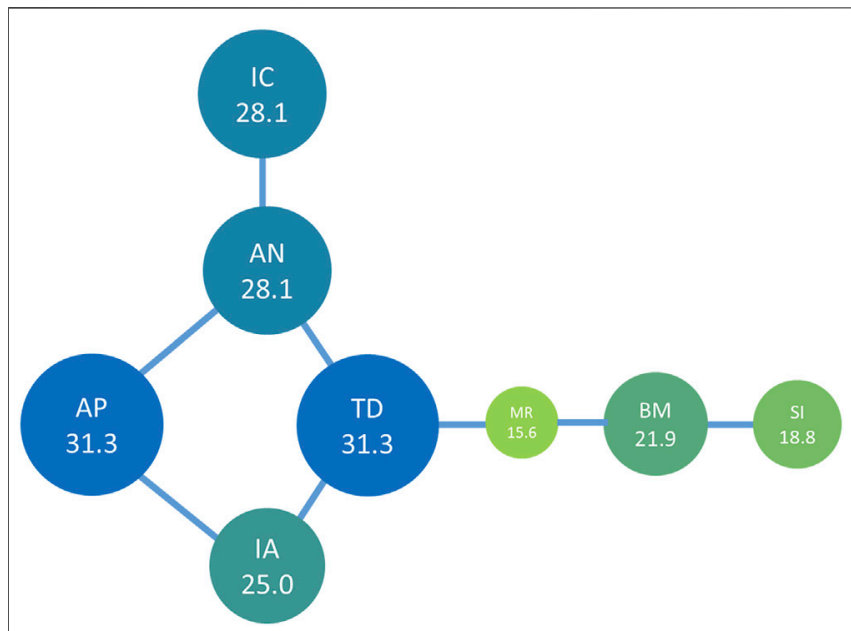

FIGURE 6 | Network analysis of the high frequency drug pairs found in Taiwanese bitter tea. The number is the UV of the plant material, and the length of the connecting line indicates co-occurrence frequency. The circle sizes represent the relative ratio of UV. AN, Ajuga nipponensis Makino; IC, Ixeris chinensis (Thunb.) Nakai; IA, Ilex asprella (Hook. \& Arn.) Champ.; TD, Tithonia diversifolia (Hemsl.) A. Gray; MR, Mallotus repandus (Willd.) Müll. Arg.; BM, Bombax malabarica DC.; SI, Solanum incanum L.; AP,

Andrographis paniculata (Burm. f.) Nees.

abundant vascular plants in Taiwan, making them very easy to obtain in the wild (Rolnik and Olas, 2021). Asteraceae plants also possess numerous and diverse flavors. The Lamiaceae family is the second largest source of plant raw materials used in Taiwanese bitter tea. Lamiaceae family plants are characterized by high amounts of volatile oils, and thus the plants are extensively used as culinary herbs (Napoli et al., 2020). Previous studies have reported that the volatile oils of these plants have antioxidant, anti-inflammatory, and antibacterial properties (Nieto, 2017), which may explain why Lamiaceae is widely used in herbal tea. This study also found several naturalized plants that were introduced from America and Africa used in bitter tea. American native plants introduced into Taiwan and used in bitter tea included Physalis angulata L., Bidens pilosa L., and Tithonia diversifolia, and African native plants included Solanum incanum L. and Momordica charantia L. (Wu et al., 2004).

The diversity analysis for the northern, central, and southern Taiwan revealed that the more the north, the more diversity of medicinal materials were. Northern Taiwan is the political and economic center of Taiwan, with very little land to grow and correct plants. The medicinal materials used in northern Taiwan are usually imported from other areas. On the other hand, the central and southern areas of Taiwan grow more plants, including medicinal plants, and have more wild land, where wild medicinal plants could be collected. Therefore, medicinal materials used by stores in the central and southern Taiwan were more readily available and more restricted locally than those in northern Taiwan. This is the reason we speculated for the more number of medicinal materials and higher diversity in Northern Taiwan than in other areas. 
All participants in the survey reported that the main function of bitter tea is to protect the liver. A previous study on green herb tea in Taiwan also reported similar results (Chen and Lin, 2012). We suspected that this result is associated with the past high prevalence of hepatitis B in Taiwan, with $15-20 \%$ of the population being chronic hepatitis $B$ carriers. Although the Taiwanese government promoted large-scale vaccination initiatives for newborns that reduced the incidence of hepatitis $\mathrm{B}$ (Wait and Chen, 2012), hepatitis C-a major cause of liver cirrhosis and liver cancer-is still prevalent in Taiwan (Yu et al., 2020). According to the cause of death statistics, chronic liver disease and liver cirrhosis remain among the top 10 causes of death, with liver cancer part of the top 10 cancers in Taiwan (Ministry of Health and Welfare, 2019). Therefore, people seek local plant materials that can "protect the liver" and provide "liver protection." Therefore, Taiwanese bitter tea may have developed as a medicinal drink under such circumstances.

Taiwanese bitter tea was mainly composed of cold and cool plant materials. According to theory of traditional Chinese medicine, cold and cool plants have a "heat clearing" effect, which can treat "heat syndrome". Heat syndrome refers to symptoms such as redness, fever, inflammation, yellow and red urine, and dry stool (Su et al., 2013). In the survey, many storekeepers mentioned that the components rendering the bitter flavor can enter the liver and that plant raw materials of bitter plants have liver "fire reduction" effects. Therefore, Taiwanese bitter tea is used to reduce "liver fire" and improve liver health. Other commonly used traditional Chinese medicines with bitter flavor for treating liver diseases include Gentiana scabra Bunge, Artemisia capillaris, and S. baicalensis (Tsai et al., 2020). In addition, bitterness-imparting compounds have been extracted from Artemisia absinthium L., a medicinal plant with hepatoprotective effects and a strong bitter taste that has been traditionally used in Europe.

During our analysis of the use and properties of Taiwanese bitter tea plant materials in the literature, we found that hepatoprotection was the fifth most published aspect for the 24 most commonly used plant materials and the fourth for the top 15 plant materials by frequency. Plants with high UV values, such as A. paniculate (Hossain et al., 2014), A. nipponensis (Hsieh et al., 2016), and T. diversifolia (Mabou Tagne et al., 2018) were reported to have hepatoprotective effects in pharmacological studies, whereas $I$. chinensis was reported to have antiviral effects against the hepatitis B virus. Meanwhile, hepatoprotection was ranked sixth for Taiwanese green herb tea. The most frequently reported pharmacological action of plant materials in bitter tea was anti-inflammation, followed by anticancer, antioxidant, antimicrobial, and anti-glycosuria effects. Long-term chronic inflammation can cause excessive oxidative stress, which is a major cause of cancer development (Bishayee, 2014); therefore, anti-inflammatories and antioxidants are considered to have potential roles in preventing cancer. For Taiwanese green herb tea, the most reported pharmacological action for the plant materials was anti-inflammation, followed by antioxidant, antimicrobial, and anticancer (Huang et al., 2020). According to these data,
Taiwanese green herb tea has similar benefits to those of bitter tea; however, bitter tea may have stronger hepatoprotective effects than does green herb tea.

A comparison of the composition of Taiwanese bitter tea with three popular herbal drinks from Lingnan (Liu Y. et al., 2013), Chaoshan (Li et al., 2017), and Fujian (Lin, 2014) revealed that $11(15.1 \%)$ plants were used in common. Moreover, there are 35 (48\%) plants used only in Taiwanese bitter tea but not used in the cool teas of the above-mentioned three areas of southern mainland China. Nevertheless, the functions of herbal teas advertised in these three regions in southern China are similar to those of herbal teas in Taiwan, all of which are used to "clear internal heat". Similarly, several medicinal plants used in herbal teas in the Lingnan, Chaoshan, and Fujian were been reported to have antioxidant, anti-inflammatory (Wu et al., 2020), and hepatoprotective (Bao et al., 2008) properties in pharmacological studies. However, given that almost half of the plant materials used in Taiwanese bitter tea are not used in herbal teas from Lingnan, Chaoshan, and Fujian, Taiwanese bitter tea can be regarded as a unique herbal tea.

The store owners selling Taiwanese bitter tea had a wide age distribution. Seventy-six percent of the source of knowledge on plant materials used in Taiwanese bitter tea was information passed down over generations. Generally, the herbal tea industry is traditional and conservative, and the recipes are often restricted within families and are not shared with external parties. Such conservative practices in the tea industry are gradually being abandoned. According to a study on herbal tea culture in Taiwan, the sale of herbal tea in Taiwan has decreased, with the number of traditional stores gradually declining (Chen and Lin, 2012). The findings of the present study can facilitate the preservation of these ancient family recipes as well as the cultural heritage of Taiwanese bitter tea through the Taiwanese government, the field of ethnopharmacology, and the food and pharmaceutical industries.

\section{CONCLUSION}

Bitter tea is a major traditional drink in Taiwan. The findings of the present study indicate that Taiwanese bitter tea is a very diverse mixture without a clear core species used in all these mixture. Moreover, the plant materials used in Taiwanese bitter tea are unique and that the constituent plants have numerous functions. More follow-up studies are required to ascertain the pharmacological effects of Taiwanese bitter tea; facilitate safer and more effective use of bitter tea in Taiwan; and preserve bitter tea as a traditional culture and resource.

\section{DATA AVAILABILITY STATEMENT}

The original contributions presented in the study are included in the article/Supplementary Material, further inquiries can be directed to the corresponding authors. 


\section{ETHICS STATEMENT}

The studies involving human participants were reviewed and approved by the CRREC-107-019. The patients/participants provided their written informed consent to participate in this study.

\section{AUTHOR CONTRIBUTIONS}

S-SH, T-YC, J-SD, L-HP, Y-CC, S-YS, and JC designed the study. S-SH, T-YC, J-SD, S-YS, and JC conducted the field work. S-SH, T-YC, L-HP, and JC performed the data analysis. S-SH, T-YC, Y-CC, and JC wrote the manuscript. All authors contributed to the article and approved the submitted version.

\section{FUNDING}

This work was supported by the Ministry of Science and Technology (grant numbers MOST 107-2320-B-039-030-MY3, MOST 107-2320B-039-063, MOST 108-2813-C-039-061-B and MOST 109-2320-

\section{REFERENCES}

Adewoye, E. O., Oguntola, M. A., and Ige, A. O. (2016). Anti-oxidative and renorestorative Effects of Physalis Angulata (Whole Plant Extract) in AlloxanInduced Diabetic Male Wistar Rats. Afr. J. Med. Med. Sci. 45 (1), 99-108.

Adisakwattana, S., Thilavech, T., and Chusak, C. (2014). Mesona Chinensis Benth Extract Prevents AGE Formation and Protein Oxidation against FructoseInduced Protein Glycation In Vitro. BMC Complement. Altern. Med. 14, 130. doi:10.1186/1472-6882-14-130

Afolayan, F. I. D., Adegbolagun, O. M., Irungu, B., Kangethe, L., Orwa, J., and Anumudu, C. I. (2016). Antimalarial Actions of Lawsonia Inermis, Tithonia Diversifolia and Chromolaena Odorata in Combination. J. Ethnopharmacol. 191, 188-194. doi:10.1016/j.jep.2016.06.045

AkyÜz, E., TÜrkoĞlu, S., SÖzgen BaŞkan, K., TÜtem, E., and Apak, M. R. (2020). Comparison of Antioxidant Capacities and Antioxidant Components of Commercial Bitter Melon (Momordica Charantia L.) Products. Turk. J. Chem. 44 (6), 1663-1673. doi:10.3906/kim-2007-67

Al-Dualimi, D. W., Shah Abdul Majid, A., Al-Shimary, S. F. F., Al-Saadi, A. A., Al Zarzour, R., Asif, M., et al. (2018). 50\% Ethanol Extract of Orthosiphon Stamineus Modulates Genotoxicity and Clastogenicity Induced by Mitomycin C. Drug Chem. Toxicol. 41 (1), 82-88. doi:10.1080/01480545.2017.1317785

Al-Emam, A., Al-Shraim, M., Eid, R., Alfaifi, M., Al-Shehri, M., Moustafa, M. F., et al. (2018). Ultrastructural Changes Induced by Solanum Incanum Aqueous Extract on HCT 116 colon Cancer Cells. Ultrastruct. Pathol. 42 (3), 255-261. doi:10.1080/01913123.2018.1447623

Alvarez, A., Pomar, F., Sevilla, M. A., and Montero, M. J. (1999). Gastric Antisecretory and Antiulcer Activities of an Ethanolic Extract of Bidens Pilosa L. Var. Radiata Schult. Bip. J. Ethnopharmacol 67 (3), 333-340. doi:10.1016/s0378-8741(99)00092-6

Alzweiri, M., Sarhan, A. A., Mansi, K., Hudaib, M., and Aburjai, T. (2011). Ethnopharmacological Survey of Medicinal Herbs in Jordan, the Northern Badia Region. J. Ethnopharmacol. 137 (1), 27-35. doi:10.1016/j.jep.2011.02.007

Arafa, A. F., Foda, D. S., Mahmoud, A. H., Metwally, N. S., and Farrag, A. R. H. (2019). Bombax ceiba Flowers Extract Ameliorates Hepatosteatosis Induced by Ethanol and Relatively Moderate Fat Diet in Rats. Toxicol. Rep. 6, 401-408. doi:10.1016/j.toxrep.2019.04.008
B-039-026); China Medical University (Grant Number CMU106N-24, CMU107-N-33, CMU109-MF-95, CMU108-MF-116, CMU108-SR-101, and CMU108-N-22); Chang Gung Memorial Hospital (Chang Gung Medical Research Program grant number CMRPF1D0123); and the Higher Education Sprout Project and Teaching Practice Research Program of China Medical University (Grant Number 1077170A) and Ministry of Education, Taiwan.

\section{ACKNOWLEDGMENTS}

The authors are grateful to the herbarium of China Medical University for providing us with a space for storing the medicinal materials collected during this study. The authors would like to thank Editage for editorial assistance.

\section{SUPPLEMENTARY MATERIAL}

The Supplementary Material for this article can be found online at: https://www.frontiersin.org/articles/10.3389/fphar.2022.816029/ full\#supplementary-material

Arantes, D. A. C., Silva, A. C. G. D., Lima, E. M., Alonso, E. C. P., Marreto, R. N., Mendonça, E. F., et al. (2021). Biological Effects of Formulation Containing Curcuminoids and Bidens Pilosa L. In Oral Carcinoma Cell Line. Braz. Oral Res. 35, e063. doi:10.1590/1807-3107bor-2021.vol35.0063

Aslam, M. S., Ahmad, M. S., Mamat, A. S., Ahmad, M. Z., and Salam, F. (2016). Antioxidant and Wound Healing Activity of Polyherbal Fractions of Clinacanthus Nutans and Elephantopus Scaber. Evid. Based Complement. Alternat. Med. 2016, 4685246. doi:10.1155/2016/4685246

Bai, M., Chen, J. J., Xu, W., Dong, S. H., Liu, Q. B., Lin, B., et al. (2020). Elephantopinolide A-P, Germacrane-type Sesquiterpene Lactones from Elephantopus Scaber Induce Apoptosis, Autophagy and G2/M Phase Arrest in Hepatocellular Carcinoma Cells. Eur. J. Med. Chem. 198, 112362. doi:10. 1016/j.ejmech.2020.112362

Bao, L., Yao, X. S., He, R. R., and Kurihara, H. (2008). [Protective Effects of Guangdong Liangcha Grandes on Restraint Stress-Induced Liver Damage in Mice]. Zhongguo Zhong Yao Za Zhi 33 (6), 664-669.

Bensky, D., Clavey, S., and Stoger, E. (2004). Chinese Herbal Medicine Materia Medica. Seattle, United States: Eastland Press.

Bhargava, S., and Shah, M. B. (2020). Evaluation of Efficacy of Bombax ceiba Extract and its Major Constituent, Mangiferin in Streptozotocin (STZ)-induced Diabetic Rats. J. Complement. Integr. Med. 18 (2), 311-318. doi:10.1515/jcim2020-0027

Bilanda, D. C., Dzeufiet, P. D. D., Kouakep, L., Aboubakar, B. F. O., Tedong, L., Kamtchouing, P., et al. (2017). Bidens Pilosa Ethylene Acetate Extract Can Protect against L-NAME-Induced Hypertension on Rats. BMC Complement. Altern. Med. 17 (1), 479. doi:10.1186/s12906-017-1972-0

Bishayee, A. (2014). The Role of Inflammation and Liver Cancer. Adv. Exp. Med. Biol. 816, 401-435. doi:10.1007/978-3-0348-0837-8_16

Boonyaketgoson, S., Rukachaisirikul, V., Phongpaichit, S., and Trisuwan, K. (2018). Naphthoquinones from the Leaves of Rhinacanthus Nasutus Having Acetylcholinesterase Inhibitory and Cytotoxic Activities. Fitoterapia 124, 206-210. doi:10.1016/j.fitote.2017.11.011

Boueroy, P., Saensa-Ard, S., Siripong, P., Kanthawong, S., and Hahnvajanawong, C. (2018). Rhinacanthin-C Extracted from Rhinacanthus Nasutus (L.) Inhibits Cholangiocarcinoma Cell Migration and Invasion by Decreasing MMP-2, uPA, FAK and MAPK Pathways. Asian Pac. J. Cancer Prev. 19 (12), 3605-3613. doi:10.31557/APJCP.2018.19.12.3605 
Brimson, J. M., Brimson, S. J., Brimson, C. A., Rakkhitawatthana, V., and Tencomnao, T. (2012). Rhinacanthus Nasutus Extracts Prevent Glutamate and Amyloid- $\beta$ Neurotoxicity in HT-22 Mouse Hippocampal Cells: Possible Active Compounds Include Lupeol, Stigmasterol and $\beta$-sitosterol. Int. J. Mol. Sci. 13 (4), 5074-5097. doi:10.3390/ijms13045074

Brimson, J. M., and Tencomnao, T. (2011). Rhinacanthus Nasutus Protects Cultured Neuronal Cells against Hypoxia Induced Cell Death. Molecules 16 (8), 6322-6338. doi:10.3390/molecules16086322

Broering, M. F., Nunes, R., De Faveri, R., De Faveri, A., Melato, J., Correa, T. P., et al. (2019). Effects of Tithonia Diversifolia (Asteraceae) Extract on Innate Inflammatory Responses. J. Ethnopharmacol. 242, 112041. doi:10.1016/j.jep. 2019.112041

Burgos, R. A., Alarcón, P., Quiroga, J., Manosalva, C., and Hancke, J. (2020). Andrographolide, an Anti-inflammatory Multitarget Drug: All Roads lead to Cellular Metabolism. Molecules 26 (1), 5. doi:10.3390/molecules26010005

Chairissy, M. D., Wulandari, L. R., and Sujuti, H. (2019). Pro-apoptotic and Antiproliferative Effects of Physalis Angulata Leaf Extract on Retinoblastoma Cells. Int. J. Ophthalmol. 12 (9), 1402-1407. doi:10.18240/ijo.2019.09.05

Chang, C. Z., Wu, S. C., Kwan, A. L., and Lin, C. L. (2016). Rhinacanthin-C, a FatSoluble Extract from Rhinacanthus Nasutus, Modulates High-Mobility Group Box 1-Related Neuro-Inflammation and Subarachnoid Hemorrhage-Induced Brain Apoptosis in a Rat Model. World Neurosurg. 86, 349-360. doi:10.1016/j. wneu.2015.08.071

Chang, C.-C., Ling, X.-H., Hsu, H.-F., Wu, J.-M., Wang, C.-P., Yang, J.-F., et al. (2016). Siegesbeckia Orientalis Extract Inhibits TGF $\beta 1$-Induced Migration and Invasion of Endometrial Cancer Cells. Molecules 21 (8), 1021. doi:10.3390/ molecules 21081021

Chang, Y. S. (2004). Investigation of the Current Status of Green Herb Stores in Taiwan. Taipei: Ministry of Health and Welfare, 337-373.

Chang, Y. S. (2005). Investigation of the Current Status of Green Herb Stores in Taiwan. Yearb. Chin. Med. Pharm.. Taipei 23 (5), 337-448.

Chao, J., Ko, C. Y., Lin, C. Y., Tomoji, M., Huang, C. H., Chiang, H. C., et al. (2020). Ethnobotanical Survey of Natural Galactagogues Prescribed in Traditional Chinese Medicine Pharmacies in Taiwan. Front. Pharmacol. 11, 625869. doi:10.3389/fphar.2020.625869

Chauhan, S., Sharma, A., Upadhyay, N. K., Singh, G., Lal, U. R., and Goyal, R. (2018). In-vitro Osteoblast Proliferation and In-Vivo Anti-osteoporotic Activity of Bombax ceiba with Quantification of Lupeol, Gallic Acid and $\beta$-sitosterol by HPTLC and HPLC. BMC Complement. Altern. Med. 18 (1), 233. doi:10.1186/ s12906-018-2299-1

Chen, C. C., Kao, C. P., Chiu, M. M., and Wang, S. H. (2017). The Anti-cancer Effects and Mechanisms of Scutellaria Barbata D. Don on CL1-5 Lung Cancer Cells. Oncotarget 8 (65), 109340-109357. doi:10.18632/oncotarget.22677

Chen, C. C., Lii, C. K., Lin, Y. H., Shie, P. H., Yang, Y. C., Huang, C. S., et al. (2020). Andrographis Paniculata Improves Insulin Resistance in High-Fat DietInduced Obese Mice and TNFa-Treated 3T3-L1 Adipocytes. Am. J. Chin. Med. 48 (5), 1073-1090. doi:10.1142/S0192415X20500524

Chen, K. H., and Lin, H. L. (2012). Herb tea Culture in Taiwan. J. Isl. Tour. Res. 5 (3), 35-73. doi:10.29859/JITR.201209.0003

Chen, L. J., Hsu, T. C., Yeh, P. J., Yow, J. L., Chang, C. L., Lin, C. H., et al. (2021). Differential Effects of Wedelia Chinensis on Human Glioblastoma Multiforme Cells. Integr. Cancer Ther. 20, 15347354211000119. doi:10.1177/ 15347354211000119

Chen, R., He, J., Tong, X., Tang, L., and Liu, M. (2016). The Hedyotis Diffusa Willd. (Rubiaceae): a Review on Phytochemistry, Pharmacology, Quality Control and Pharmacokinetics. Molecules 21 (6), 710. doi:10.3390/molecules21060710

Chiang, L. C., Cheng, H. Y., Chen, C. C., and Lin, C. C. (2004). In Vitro antileukemic and Antiviral Activities of Traditionally Used Medicinal Plants in Taiwan. Am. J. Chin. Med. 32 (5), 695-704. doi:10.1142/S0192415X04002284

Chiavari-Frederico, M. O., Barbosa, L. N., Carvalho Dos Santos, I., Ratti da Silva, G., Fernandes de Castro, A., de Campos Bortolucci, W., et al. (2020). Antimicrobial Activity of Asteraceae Species against Bacterial Pathogens Isolated from Postmenopausal Women. PLoS One 15 (1), e0227023. doi:10. 1371/journal.pone.0227023

Chien, S. C., Young, P. H., Hsu, Y. J., Chen, C. H., Tien, Y. J., Shiu, S. Y., et al. (2009). Anti-diabetic Properties of Three Common Bidens Pilosa Variants in Taiwan. Phytochemistry 70 (10), 1246-1254. doi:10.1016/j.phytochem.2009.07.011
Chu, J. M. T., Xiong, W., Linghu, K. G., Liu, Y., Zhang, Y., Zhao, G. D., et al. (2018). Siegesbeckia Orientalis L. Extract Attenuates Postoperative Cognitive Dysfunction, Systemic Inflammation, and Neuroinflammation. Exp. Neurobiol. 27 (6), 564-573. doi:10.5607/en.2018.27.6.564

Chuang, K. A., Li, M. H., Lin, N. H., Chang, C. H., Lu, I. H., Pan, I. H., et al. (2017). Rhinacanthin C Alleviates Amyloid-Beta Fibrils' Toxicity on Neurons and Attenuates Neuroinflammation Triggered by Lps, Amyloid-Beta, and Interferon-Gamma in Glial Cells. Oxid. Med. Cel Longev. 2017, 5414297. doi: $10.1155 / 2017 / 5414297$

Chung, T. W., Choi, H., Lee, J. M., Ha, S. H., Kwak, C. H., Abekura, F., et al. (20172017). Corrigendum to "Oldenlandia Diffusa Suppresses Metastatic Potential through Inhibiting Matrix Metalloproteinase-9 and Intercellular Adhesion Molecule-1 Expression via P38 and ERK1/2 MAPK Pathways and Induces Apoptosis in Human Breast Cancer MCF-7 Cells" [J. Ethnopharmacol. 195 (2017) 309-317]. J. Ethnopharmacol. 204, 309189-309317. doi:10.1016/j. jep.2017.04.016

Chusak, C., Thilavech, T., and Adisakwattana, S. (2014). Consumption of Mesona Chinensis Attenuates Postprandial Glucose and Improves Antioxidant Status Induced by a High Carbohydrate Meal in Overweight Subjects. Am. J. Chin. Med. 42 (2), 315-336. doi:10.1142/S0192415X14500219

Da Silva, B. J. M., Pereira, S. W. G., Rodrigues, A. P. D., Do Nascimento, J. L. M., and Silva, E. O. (2018). In Vitro antileishmanial Effects of Physalis Angulata Root Extract on Leishmania Infantum. J. Integr. Med. 16 (6), 404-410. doi:10. 1016/j.joim.2018.08.004

Dai, W. P., Li, G., Li, X., Hu, Q. P., Liu, J. X., Zhang, F. X., et al. (2014). The Roots of Ilex Asprella Extract Lessens Acute Respiratory Distress Syndrome in Mice Induced by Influenza Virus. J. Ethnopharmacol. 155 (3), 1575-1582. doi:10. 1016/j.jep.2014.07.051

Damsud, T., Grace, M. H., Adisakwattana, S., and Phuwapraisirisan, P. (2014). Orthosiphol A from the Aerial Parts of Orthosiphon Aristatus Is Putatively Responsible for Hypoglycemic Effect via Alpha-Glucosidase Inhibition. Nat. Prod. Commun. 9 (5), 639-641. doi:10.1177/1934578x1400900512

Darah, I., Lim, S. H., and Nithianantham, K. (2013). Effects of Methanol Extract of Wedelia Chinensis Osbeck (Asteraceae) Leaves against Pathogenic Bacteria with Emphasise on Bacillus Cereus. Indian J. Pharm. Sci. 75 (5), 533-539.

Ehigie, A. F., Wei, P., Wei, T., Yan, X., Olorunsogo, O. O., Ojeniyi, F. D., et al. (2021). Momordica Charantia L. Induces Non-apoptotic Cell Death in Human MDA-MB-436 Breast and A549 Lung Cancer Cells by Disrupting Energy Metabolism and Exacerbating Reactive Oxygen Species' Generation. J. Ethnopharmacol. 277, 114036. doi:10.1016/j.jep.2021.114036

Ejelonu, O. C., Elekofehinti, O. O., and Adanlawo, I. G. (2017). Tithonia Diversifolia Saponin-Blood Lipid Interaction and its Influence on Immune System of normal Wistar Rats. Biomed. Pharmacother. 87, 589-595. doi:10. 1016/j.biopha.2017.01.017

Engels, N. S., Gierlikowska, B., Waltenberger, B., Chang, F. R., Kiss, A. K., and Stuppner, H. (2020). A New Diterpene and Anti-inflammatory Sesquiterpene Lactones from Sigesbeckia Orientalis. Planta Med. 86 (15), 1108-1117. doi:10. 1055/a-1232-6869

Fan, M., Lee, J. I., Ryu, Y. B., Choi, Y. J., Tang, Y., Oh, M., et al. (2021). Comparative Analysis of Metabolite Profiling of Momordica Charantia Leaf and the Antiobesity Effect through Regulating Lipid Metabolism. Int. J. Environ. Res. Public Health 18 (11), 5584. doi:10.3390/ijerph18115584

Ferreira Farias, A. L., Lobato Rodrigues, A. B., Lopes Martins, R., de Menezes Rabelo, É., Ferreira Farias, C. W., and Moreira da Silva de Almeida, S. S. (2019). Chemical Characterization, Antioxidant, Cytotoxic and Microbiological Activities of the Essential Oil of Leaf of Tithonia Diversifolia (Hemsl) A. Gray (Asteraceae). Pharmaceuticals (Basel) 12 (1), 34. doi:10.3390/ph12010034

Fouladi, S., Masjedi, M., Ganjalikhani Hakemi, M., and Eskandari, N. (2019). The Review of In Vitro and In Vivo Studies over the Glycyrrhizic Acid as Natural Remedy Option for Treatment of Allergic Asthma. Iran J. Allergy Asthma Immunol. 18 (1), 1-11. doi:10.18502/ijaai.v18i1.626

Fu, L., Pei, D., Yu, M., Shang, H., Si, J. G., Zhang, H. W., et al. (2020). New Phenolic Acids from the Whole Herb of Elephantopus Scaber Linn. And Their Antiinflammatory Activity. Nat. Prod. Res. 35, 1-8. doi:10.1080/14786419.2020. 1723086

Fu, Y., Yang, J. C., Cunningham, A. B., Towns, A. M., Zhang, Y., Yang, H. Y., et al. (2018). A Billion Cups: the Diversity, Traditional Uses, Safety Issues and 
Potential of Chinese Herbal Teas. J. Ethnopharmacol. 222, 217-228. doi:10. 1016/j.jep.2018.04.026

Gao, J., Lu, W. F., Dai, Z. J., Lin, S., Zhao, Y., Li, S., et al. (2014). Induction of Apoptosis by Total Flavonoids from Scutellaria Barbata D. Don in Human Hepatocarcinoma MHCC97-H Cells via the Mitochondrial Pathway. Tumour Biol. 35 (3), 2549-2559. doi:10.1007/s13277-013-1336-4

Gertsch, J. (2011). Botanical Drugs, Synergy, and Network Pharmacology: Forth and Back to Intelligent Mixtures. Planta Med. 77 (11), 1086-1098. doi:10.1055/ s-0030-1270904

Gras, A., Parada, M., Rigat, M., Vallès, J., and Garnatje, T. (2018). Folk Medicinal Plant Mixtures: Establishing a Protocol for Further Studies. J. Ethnopharmacol. 214, 244-273. doi:10.1016/j.jep.2017.12.014

Güneş, S., Savran, A., Paksoy, M. Y., Koşar, M., and Çakılcıŏlu, U. (2017). Ethnopharmacological Survey of Medicinal Plants in Karaisalı and its Surrounding (Adana-Turkey). J. Herb. Med. 8, 68-75. doi:10.1016/j.hermed. 2017.04.002

Gupta, P., Goyal, R., Chauhan, Y., and Sharma, P. L. (2013). Possible Modulation of FAS and PTP-1B Signaling in Ameliorative Potential of Bombax ceiba against High Fat Diet Induced Obesity. BMC Complement. Altern. Med. 13, 281. doi:10. $1186 / 1472-6882-13-281$

Hasan, M. M., Uddin, N., Hasan, M. R., Islam, A. F., Hossain, M. M., Rahman, A. B., et al. (2014). Analgesic and Anti-inflammatory Activities of Leaf Extract of Mallotus Repandus (Willd.) Muell. Arg. Biomed. Res. Int. 2014, 539807. doi:10. $1155 / 2014 / 539807$

Hassan, W. R. M., Basir, R., Ali, A. H., Embi, N., and Sidek, H. M. (2019). Antimalarial and Cytokine-Modulating Effects of Andrographolide in a Murine Model of Malarial Infection. Trop. Biomed. 36 (3), 776-791.

Hiradeve, S. M., and Rangari, V. D. (2014). A Review on Pharmacology and Toxicology of Elephantopus Scaber Linn. Nat. Prod. Res. 28 (11), 819-830. doi:10.1080/14786419.2014.883394

Hong, M., Fan, X., Liang, S., Xiang, W., Chen, L., Yang, Y., et al. (2021). Total Flavonoids of Bidens Pilosa Ameliorates Bone Destruction in Collagen-Induced Arthritis. Planta Med. 87, 550-559. doi:10.1055/a-1352-5124

Horii, H., Suzuki, R., Sakagami, H., Umemura, N., Ueda, J. Y., and Shirataki, Y. (2012). Induction of Non-apoptotic Cell Death in Human Oral Squamous Cell Carcinoma Cell Lines by Rhinacanthus Nasutus Extract. In Vivo 26 (2), 305-309.

Horiuchi, M., Wachi, H., and Seyama, Y. (2010). Effects of Bidens Pilosa L. Var. Radiata Scherff on Experimental Gastric Lesion. J. Nat. Med. 64 (4), 430-435. doi:10.1007/s11418-010-0426-5

Hossain, M. S., Urbi, Z., Sule, A., and Hafizur Rahman, K. M. (2014). Andrographis Paniculata (Burm. f.) Wall. Ex Nees: a Review of Ethnobotany, Phytochemistry, and Pharmacology. ScientificWorldJournal 2014, 274905. doi:10.1155/2014/ 274905

Hossain, S., Urbi, Z., Karuniawati, H., Mohiuddin, R. B., Moh Qrimida, A., Allzrag, A. M. M., et al. (2021). Andrographis Paniculata (Burm. f.) Wall. Ex Nees: an Updated Review of Phytochemistry, Antimicrobial Pharmacology, and Clinical Safety and Efficacy. Life (Basel) 11 (4), 348. doi:10.3390/ life1 1040348

Hsiao, C. Y., Chen, Y. M., Hsu, Y. J., Huang, C. C., Sung, H. C., and Chen, S. S. (2017). Supplementation with Hualian No. 4 Wild Bitter Gourd (Momordica Charantia Linn. Var. Abbreviata ser.) Extract Increases Anti-fatigue Activities and Enhances Exercise Performance in Mice. J. Vet. Med. Sci. 79 (6), 1110-1119. doi:10.1292/jvms.17-0079

Hsieh, C. W., Ko, W. C., Ho, W. J., Chang, C. K., Chen, G. J., and Tsai, J. C. (2016). Antioxidant and Hepatoprotective Effects of Ajuga Nipponensis Extract by Ultrasonic-Assisted Extraction. Asian Pac. J. Trop. Med. 9 (5), 420-425. doi:10. 1016/j.apjtm.2016.03.029

Hsieh, C. Y., Lin, Y. T., Huang, S. S., Hsu, P. C., Lu, F. L., Tsai, C. F., et al. (2017). Analysis of the Confusion and Misuse of Chinese Herbs in the Registration of Chinese Medicinal Preparations. Ann. Rept. Food Drug Res. 8, 103-116.

Hsu, C. L., Hong, B. H., Yu, Y. S., and Yen, G. C. (2010). Antioxidant and Antiinflammatory Effects of Orthosiphon Aristatus and its Bioactive Compounds. J. Agric. Food Chem. 58 (4), 2150-2156. doi:10.1021/jf903557c

Hu, S. Y. (2005). Food Plants of China. Hong Kong: The Chinese University Press, $3-46 \mathrm{p}$.

Hu, X. Y., Shu, X. C., Guo, Y., and Ma, Y. (2012). Effect of an Ilex Asprella Root Decoction on the Related Genes of Lipid Metabolism from Chronic Stress and Hyperlipidemic Fatty Liver in Rats. Chin. Med. J. (Engl) 125 (19), 3539-3542.
Huang, L., Huang, M., Shen, M., Wen, P., Wu, T., Hong, Y., et al. (2019). Sulfated Modification Enhanced the Antioxidant Activity of Mesona Chinensis Benth Polysaccharide and its Protective Effect on Cellular Oxidative Stress. Int. J. Biol. Macromol. 136, 1000-1006. doi:10.1016/j.ijbiomac.2019.06.199

Huang, L., Shen, M., Wu, T., Yu, Y., Yu, Q., Chen, Y., et al. (2020). Mesona Chinensis Benth Polysaccharides Protect against Oxidative Stress and Immunosuppression in Cyclophosphamide-Treated Mice via MAPKs Signal Transduction Pathways. Int. J. Biol. Macromol. 152, 766-774. doi:10.1016/j. ijbiomac.2020.02.318

Huang, S. S., Chen, T. Y., Deng, J. S., Pao, L. H., Cheng, Y. C., and Chao, J. (2020). An Ethnobotanical Study on Qing-Căo-Chá Tea in Taiwan. Front. Pharmacol. 11, 931. doi:10.3389/fphar.2020.00931

Huang, W., Liang, Y., Wang, J., Li, G., Wang, G., Li, Y., et al. (2016). Antiangiogenic Activity and Mechanism of Kaurane Diterpenoids from Wedelia Chinensis. Phytomedicine 23 (3), 283-292. doi:10.1016/j.phymed.2015. 12.021

Ide, M., Yoshida, I., Kumagai, M., Mishima, T., Takahashi, Y., Fujita, K., et al. (2020). Tithonia Diversifolia-derived Orizabin Suppresses Cell Adhesion, Differentiation, and Oxidized LDL Accumulation by Akt Signaling Suppression via PTEN Promotion in THP-1 Cells. J. Food Biochem. 44 (7), e13268. doi:10.1111/jfbc. 13268

Jadhav, A. K., and Karuppayil, S. M. (2021). Andrographis Paniculata (Burm. F) Wall Ex Nees: Antiviral Properties. Phytother. Res. 35 (10), 5365-5373. doi:10. $1002 /$ ptr.7145

Jeenkeawpieam, J., Yodkeeree, S., Andrianopoulos, A., Roytrakul, S., and Pongpom, M. (2020). Antifungal Activity and Molecular Mechanisms of Partial Purified Antifungal Proteins from Rhinacanthus Nasutus against Talaromyces marneffei. J. Fungi (Basel) 6 (4), 333. doi:10.3390/jof6040333

Jiang, M., Zhao, S., Yang, S., Lin, X., He, X., Wei, X., et al. (2020). An "essential Herbal Medicine"-Licorice: A Review of Phytochemicals and its Effects in Combination Preparations. J. Ethnopharmacol. 249, 112439. doi:10.1016/j.jep. 2019.112439

Jin, B., Liu, Y., Xie, J., Luo, B., and Long, C. (2018). Ethnobotanical Survey of Plant Species for Herbal tea in a Yao Autonomous County (Jianghua, China): Results of a 2-year Study of Traditional Medicinal Markets on the Dragon Boat Festival. J. Ethnobiol. Ethnomed 14 (1), 58. doi:10.1186/s13002-018-0257-0

Jin, Y., Chen, W., Yang, H., Yan, Z., Lai, Z., Feng, J., et al. (2017). Scutellaria Barbata D. Don Inhibits Migration and Invasion of Colorectal Cancer Cells via Suppression of PI3K/AKT and TGF- $\beta /$ Smad Signaling Pathways. Exp. Ther. Med. 14 (6), 5527-5534. doi:10.3892/etm.2017.5242

Joubert, E., Gelderblom, W. C., Louw, A., and de Beer, D. (2008). South African Herbal Teas: Aspalathus Linearis, Cyclopia Spp. And Athrixia Phylicoides-Aa Review. J. Ethnopharmacol 119 (3), 376-412. doi:10.1016/j. jep.2008.06.014

Kan, X., Zhang, W., You, R., Niu, Y., Guo, J., and Xue, J. (2017). Scutellaria Barbata D. Don Extract Inhibits the Tumor Growth through Down-Regulating of Treg Cells and Manipulating Th1/Th17 Immune Response in Hepatoma H22Bearing Mice. BMC Complement. Altern. Med. 17 (1), 41. doi:10.1186/ s12906-016-1551-9

Kernan, M. R., Sendl, A., Chen, J. L., Jolad, S. D., Blanc, P., Murphy, J. T., et al. (1997). Two New Lignans with Activity against Influenza Virus from the Medicinal Plant Rhinacanthus Nasutus. J. Nat. Prod. 60 (6), 635-637. doi:10. 1021/np960613i

Kim, J. W., Kim, H. P., and Sung, S. H. (2017). Cytotoxic Pterosins from Pteris Multifida Roots against HCT116 Human colon Cancer Cells. Bioorg. Med. Chem. Lett. 27 (14), 3144-3147. doi:10.1016/j.bmcl.2017.05.034

Kim, J. W., Seo, J. Y., Oh, W. K., and Sung, S. H. (2016). Anti-neuroinflammatory Ent-Kaurane Diterpenoids from Pteris Multifida Roots. Molecules 22 (1), 27. doi:10.3390/molecules22010027

Kim, K. W., Jin, U. H., Kim, D. I., Lee, T. K., Kim, M. S., Oh, M. J., et al. (2008). Antiproliferative Effect of Scutellaria Barbata D. Don. On Cultured Human Uterine Leiomyoma Cells by Down-Regulation of the Expression of Bcl-2 Protein. Phytother. Res. 22 (5), 583-590. doi:10.1002/ptr.1996

Komati, A., Anand, A., Shaik, H., Mudiam, M. K. R., Suresh Babu, K., and Tiwari, A. K. (2020). Bombax ceiba (Linn.) Calyxes Ameliorate Methylglyoxal-Induced Oxidative Stress via Modulation of RAGE Expression: Identification of Active Phytometabolites by GC-MS Analysis. Food Funct. 11 (6), 5486-5497. doi:10. 1039/c9fo02714a 
Kulkarni, P., Lohidasan, S., and Mahadik, K. (2021). Bioanalytical Method Development for Momordicinin and its Application to Long-Term Pharmacokinetics in Diabetic Rats. Drug Dev. Ind. Pharm. 47, 1064-1071. doi:10.1080/03639045.2021.1908337

Kupradinun, P., Siripong, P., Chanpai, R., Piyaviriyagul, S., Rungsipipat, A., and Wangnaitham, S. (2009). Effects of Rhinacanthus Nasutus Kurz on colon Carcinogenesis in Mice. Asian Pac. J. Cancer Prev. 10 (1), 103-106.

Kwak, H. J., Park, S., Kim, N., Yoo, G., Park, J. H., Oh, Y., et al. (2018). Neuraminidase Inhibitory Activity by Compounds Isolated from Aerial Parts of Rhinacanthus Nasutus. Nat. Prod. Res. 32 (17), 2111-2115. doi:10. 1080/14786419.2017.1365067

Lashin, I., Fouda, A., Gobouri, A. A., Azab, E., Mohammedsaleh, Z. M., and Makharita, R. R. (2021). Antimicrobial and In Vitro Cytotoxic Efficacy of Biogenic Silver Nanoparticles (Ag-NPs) Fabricated by Callus Extract of Solanum Incanum L. Biomolecules 11 (3), 341. doi:10.3390/biom11030341

Lee, D. S., Keo, S., Cheng, S. K., Oh, H., and Kim, Y. C. (2017). Protective Effects of Cambodian Medicinal Plants on Tert-butyl H-ydroperoxide-induced $\mathrm{H}$-epatotoxicity via Nrf2-mediated H-eme O-xygenase-1. Mol. Med. Rep. 15 (1), 451-459. doi:10.3892/mmr.2016.6011

Lee, H. J., Choi, E. J., Park, S., and Lee, J. J. (2020). Laxative and Antioxidant Effects of Ramie (Boehmeria Nivea L.) Leaf Extract in Experimental Constipated Rats. Food Sci. Nutr. 8 (7), 3389-3401. doi:10.1002/fsn3.1619

Li, D. L., Zheng, X. L., Duan, L., Deng, S. W., Ye, W., Wang, A. H., et al. (2017). Ethnobotanical Survey of Herbal tea Plants from the Traditional Markets in Chaoshan, China. J. Ethnopharmacol. 205, 195-206. doi:10.1016/j.jep.2017. 02.040

Li, H., Xie, W., Qiao, X., Cui, H., Yang, X., and Xue, C. (2020). Structural Characterization of Arabinogalactan Extracted from Ixeris Chinensis (Thunb.) Nakai and its Immunomodulatory Effect on RAW264.7 Macrophages. Int. J. Biol. Macromol. 143, 977-983. doi:10.1016/j.ijbiomac. 2019.09.158

Li, Q., Hao, Z., Hong, Y., He, W., and Zhao, W. (2018). Reprogramming Tumor Associated Macrophage Phenotype by a Polysaccharide from Ilex Asprella for Sarcoma Immunotherapy. Int. J. Mol. Sci. 19 (12), 3816. doi:10.3390/ ijms 19123816

Lim, J. Y., Lee, J. H., Lee, B. R., Kim, M. A., Lee, Y. M., Kim, D. K., et al. (2020). Extract of Boehmeria Nivea Suppresses Mast Cell-Mediated Allergic Inflammation by Inhibiting Mitogen-Activated Protein Kinase and Nuclear Factor-Kb. Molecules 25 (18), 4178. doi:10.3390/molecules25184178

Lin, F. M., Chen, L. R., Lin, E. H., Ke, F. C., Chen, H. Y., Tsai, M. J., et al. (2007). Compounds from Wedelia Chinensis Synergistically Suppress Androgen Activity and Growth in Prostate Cancer Cells. Carcinogenesis 28 (12), 2521-2529. doi:10.1093/carcin/bgm137

Lin, J., Feng, J., Yang, H., Yan, Z., Li, Q., Wei, L., et al. (2017). Scutellaria Barbata D. Don Inhibits 5-fluorouracil Resistance in Colorectal Cancer by Regulating PI3K/AKT Pathway. Oncol. Rep. 38 (4), 2293-2300. doi:10.3892/or.2017. 5892

Lin, J. M., Lin, C. C., Chen, M. F., Ujiie, T., and Takada, A. (1995). Scavenging Effects of Mallotus Repandus on Active Oxygen Species. J. Ethnopharmacol. 46 (3), 175-181. doi:10.1016/0378-8741(95)01246-a

Lin, K. H., Marthandam Asokan, S., Kuo, W. W., Hsieh, Y. L., Lii, C. K., Viswanadha, V., et al. (2020). Andrographolide Mitigates Cardiac Apoptosis to Provide Cardio-protection in High-Fat-Diet-Induced Obese Mice. Environ. Toxicol. 35 (6), 707-713. doi:10.1002/tox.22906

Lin, R. S. (2014). Study on the Medicinal Value and Resources of Fujian Local Herbal Tea Plants. Fujian: Fujian Agriculture and Forestry University. Fujian Agriculture and Forestry University.

Lin, W. L., Wang, S. M., Ho, Y. J., Kuo, H. C., Lee, Y. J., and Tseng, T. H. (2014). Ethyl Acetate Extract of Wedelia Chinensis Inhibits Tert-Butyl Hydroperoxide-Induced Damage in PC12 Cells and D-Galactose-Induced Neuronal Cell Loss in Mice. BMC Complement. Altern. Med. 14, 491. doi:10. 1186/1472-6882-14-491

Liu, H. L., Kao, T. H., Shiau, C. Y., and Chen, B. H. (2018). Functional Components in Scutellaria Barbata D. Don with Anti-inflammatory Activity on RAW 264.7 Cells. J. Food Drug Anal. 26 (1), 31-40. doi:10.1016/j.jfda.2016.11.022

Liu, J., Feng, W., and Peng, C. (2020). A Song of Ice and Fire: Cold and Hot Properties of Traditional Chinese Medicines. Front. Pharmacol. 11, 598744. doi:10.3389/fphar.2020.598744
Liu, M., Wang, W., Li, X., Shi, D., Mei, H., Jin, X., et al. (2013). Wedelia Chinensis Inhibits Nasopharyngeal Carcinoma CNE-1 Cell Growth by Inducing G2/M Arrest in a Chk1-dependent Pathway. Am. J. Chin. Med. 41 (5), 1153-1168. doi:10.1142/S0192415X1350078X

Liu, S., Lin, L., Shen, M., Wang, W., Xiao, Y., and Xie, J. (2018). Effect of Mesona Chinensis Polysaccharide on the Pasting, thermal and Rheological Properties of Wheat Starch. Int. J. Biol. Macromol. 118 (Pt A), 945-951. doi:10.1016/j. ijbiomac.2018.06.178

Liu, W., Fan, T., Li, M., Zhang, G., Guo, W., Yang, X., et al. (2020). Andrographolide Potentiates PD-1 Blockade Immunotherapy by Inhibiting COX2-Mediated PGE2 Release. Int. Immunopharmacol. 81, 106206. doi:10. 1016/j.intimp.2020.106206

Liu, Y., Ahmed, S., and Long, C. (2013). Ethnobotanical Survey of Cooling Herbal Drinks from Southern China. J. Ethnobiol. Ethnomed. 9, 82. doi:10.1186/17464269-9-82

Lu, F. J., Ding, L. Q., Cao, S. J., Zhang, D. Q., Zhang, B. L., and Qiu, F. (2018). [Chemistry and Biology Research on Bitter-Taste Chinese Materia Medica with Function of Regulating Glycolipid Metabolism]. Zhongguo Zhong Yao Za Zhi 43 (19), 3834-3840. doi:10.19540/j.cnki.cjcmm.20180709.006

Lu, J., Ma, Y., Wu, J., Huang, H., Wang, X., Chen, Z., et al. (2019). A Review for the Neuroprotective Effects of Andrographolide in the central Nervous System. Biomed. Pharmacother. 117, 109078. doi:10.1016/j.biopha.2019. 109078

Lu, K. H., Chang, Y. F., Yin, P. H., Chen, T. T., Ho, Y. L., Chang, Y. S., et al. (2010). In Vitro and In Vivo Apoptosis-Inducing Antileukemic Effects of Mucuna Macrocarpa Stem Extract on HL-60 Human Leukemia Cells. Integr. Cancer Ther. 9 (3), 298-308. doi:10.1177/1534735410378661

Lu, M. R., Huang, H. L., Chiou, W. F., and Huang, R. L. (2017). Induction of Apoptosis by Tithonia Diversifolia in Human Hepatoma Cells. Pharmacogn. Mag. 13 (52), 702-706. doi:10.4103/0973-1296.218113

Ma, T., Zhang, Y., Zhang, C., Luo, J. G., and Kong, L. Y. (2017). Downregulation of TIGAR Sensitizes the Antitumor Effect of Physapubenolide through Increasing Intracellular ROS Levels to Trigger Apoptosis and Autophagosome Formation in Human Breast Carcinoma Cells. Biochem. Pharmacol. 143, 90-106. doi:10. 1016/j.bcp.2017.07.018

Mabou Tagne, A., Marino, F., and Cosentino, M. (2018). Tithonia Diversifolia (Hemsl.) A. Gray as a Medicinal Plant: a Comprehensive Review of its Ethnopharmacology, Phytochemistry, Pharmacotoxicology and Clinical Relevance. J. Ethnopharmacol. 220, 94-116. doi:10.1016/j.jep.2018.03.025

Malik, Z., Parveen, R., Parveen, B., Zahiruddin, S., Aasif Khan, M., Khan, A., et al. (2021). Anticancer Potential of Andrographolide from Andrographis Paniculata (Burm.f.) Nees and its Mechanisms of Action. J. Ethnopharmacol. 272, 113936. doi:10.1016/j.jep.2021.113936

Manjamalai, A., and Berlin Grace, V. M. (2012). Antioxidant Activity of Essential Oils from Wedelia Chinensis (Osbeck) In Vitro and In Vivo Lung Cancer Bearing C57BL/6 Mice. Asian Pac. J. Cancer Prev. 13 (7), 3065-3071. doi:10. 7314/apjcp.2012.13.7.3065

Marconett, C. N., Morgenstern, T. J., San Roman, A. K., Sundar, S. N., Singhal, A. K., and Firestone, G. L. (2010). BZL101, a Phytochemical Extract from the Scutellaria Barbata Plant, Disrupts Proliferation of Human Breast and Prostate Cancer Cells through Distinct Mechanisms Dependent on the Cancer Cell Phenotype. Cancer Biol. Ther. 10 (4), 397-405. doi:10.4161/cbt.10.4.12424

Maregesi, S., van Miert, S., Pannecouque, C., Feiz Haddad, M. H., Hermans, N., Wright, C. W., et al. (2010). Screening of Tanzanian Medicinal Plants against Plasmodium Falciparum and Human Immunodeficiency Virus. Planta Med. 76 (2), 195-201. doi:10.1055/s-0029-1186024

Matsubara, T., Bohgaki, T., Watarai, M., Suzuki, H., Ohashi, K., and Shibuya, H. (1999). Antihypertensive Actions of Methylripariochromene A from Orthosiphon Aristatus, an Indonesian Traditional Medicinal Plant. Biol. Pharm. Bull. 22 (10), 1083-1088. doi:10.1248/bpb.22.1083

Matsumoto, T., Horiuchi, M., Kamata, K., and Seyama, Y. (2009). Effects of Bidens Pilosa L. Var. Radiata SCHERFF Treated with Enzyme on Histamine-Induced Contraction of guinea Pig Ileum and on Histamine Release from Mast Cells. J. Smooth Muscle Res. 45 (2-3), 75-86. doi:10.1540/jsmr.45.75

Mei, X. Y., Zhou, L. Y., Zhang, T. Y., Lu, B., and Ji, L. L. (2017). Scutellaria Barbata Attenuates Diabetic Retinopathy by Preventing Retinal Inflammation and the Decreased Expression of Tight junction Protein. Int. J. Ophthalmol. 10 (6), 870-877. doi:10.18240/ijo.2017.06.07 
Meira, C. S., Guimarães, E. T., Dos Santos, J. A., Moreira, D. R., Nogueira, R. C., Tomassini, T. C., et al. (2015). In Vitro and In Vivo Antiparasitic Activity of Physalis Angulata L. Concentrated Ethanolic Extract against Trypanosoma Cruzi. Phytomedicine 22 (11), 969-974. doi:10.1016/j.phymed.2015.07.004

Meng, F., Li, Q., Qi, Y., He, C., Wang, C., and Zhang, Q. (2018). Characterization and Immunoregulatory Activity of Two Polysaccharides from the Root of Ilex Asprella. Carbohydr. Polym. 197, 9-16. doi:10.1016/j.carbpol.2018.05.066

Ministry of Health and Welfare (2019). Analysis on Statistical Results of Death Causes in 2019. Available at: https://dep.mohw.gov.tw/DOS/cp-4927-54466113.html (Accessed June 16, 2020).

Ministry of Health and Welfare (2021). The Illustration Database of Common Medicinal Plants in Taiwan. Available at: https://www.cmtcmp.mohw.gov.tw/ INDEX_ENG.ASPX (accessed December 31, 2021).

Miura, T., Nosaka, K., Ishii, H., and Ishida, T. (2005). Antidiabetic Effect of Nitobegiku, the Herb Tithonia Diversifolia, in KK-Ay Diabetic Mice. Biol. Pharm. Bull. 28 (11), 2152-2154. doi:10.1248/bpb.28.2152

Mondal, M., Hossain, M. M., Hasan, M. R., Tarun, M. T. I., Islam, M. A. F., Choudhuri, M. S. K., et al. (2020). Hepatoprotective and Antioxidant Capacity of Mallotus Repandus Ethyl Acetate Stem Extract against D-GalactosamineInduced Hepatotoxicity in Rats. ACS Omega 5 (12), 6523-6531. doi:10.1021/ acsomega.9b04189

Mussard, E., Jousselin, S., Cesaro, A., Legrain, B., Lespessailles, E., Esteve, E., et al. (2020). Andrographis Paniculata and its Bioactive Diterpenoids against Inflammation and Oxidative Stress in Keratinocytes. Antioxidants (Basel) 9 (6), 530. doi:10.3390/antiox9060530

Nadia, N. A. C., Cédric, Y., Raoul, S. N. S., Christian, N. O., Azizi, M. A., Diane, G. D. C., et al. (2020). Antimalarial Activity of Ethyl Acetate Extract and Fraction of Bidens Pilosa against Plasmodium Berghei (ANKA). J. Parasitol. Res. 2020, 8832724. doi:10.1155/2020/8832724

Napoli, E., Siracusa, L., and Ruberto, G. (2020). New Tricks for Old Guys: Recent Developments in the Chemistry, Biochemistry, Applications and Exploitation of Selected Species from the Lamiaceae Family. Chem. Biodivers. 17 (3), e1900677. doi:10.1002/cbdv.201900677

Nayak, A. G., Kumar, N., Shenoy, S., and Roche, M. (2021). Evaluation of the merit of the Methanolic Extract of Andrographis Paniculata to Supplement Antisnake Venom in Reversing Secondary Hemostatic Abnormalities Induced by Naja naja Venom. 3 Biotech. 11 (5), 228. doi:10.1007/s13205-021-02766-z

Ngoc, T. M., Phuong, N. T. T., Khoi, N. M., Park, S., Kwak, H. J., Nhiem, N. X., et al. (2019). A New Naphthoquinone Analogue and Antiviral Constituents from the Root of Rhinacanthus Nasutus. Nat. Prod. Res. 33 (3), 360-366. doi:10.1080/ 14786419.2018.1452004

Nguepi, I. S. T., Ngueguim, F. T., Gounoue, R. K., Mbatchou, A., and Dimo, T. (2021). Curative Effects of the Aqueous Extract of Tithonia Diversifolia (Hemsl.) A. gray (Asteraceae) against Ethanol Induced-Hepatotoxicity in Rats. J. Basic Clin. Physiol. Pharmacol. 32 (6), 1137-1143. doi:10.1515/ jbcpp-2019-0370

Nguyen, T. D., Thuong, P. T., Hwang, I. H., Hoang, T. K., Nguyen, M. K., Nguyen, H. A., et al. (2017). Anti-hyperuricemic, Anti-inflammatory and Analgesic Effects of Siegesbeckia Orientalis L. Resulting from the Fraction with High Phenolic Content. BMC Complement. Altern. Med. 17 (1), 191. doi:10.1186/ s12906-017-1698-z

Nieto, G. (2017). Biological Activities of Three Essential Oils of the Lamiaceae Family. Medicines 4 (3), 63. doi:10.3390/medicines 4030063

Ohashi, K., Bohgaki, T., and Shibuya, H. (2000). [Antihypertensive Substance in the Leaves of Kumis Kucing (Orthosiphon Aristatus) in Java Island]. Yakugaku Zasshi 120 (5), 474-482. doi:10.1248/yakushi1947.120.5_474

Owoyele, V. B., Wuraola, C. O., Soladoye, A. O., and Olaleye, S. B. (2004). Studies on the Anti-inflammatory and Analgesic Properties of Tithonia Diversifolia Leaf Extract. J. Ethnopharmacol. 90 (2-3), 317-321. doi:10.1016/j.jep.2003. 10.010

Ozmen, A., Madlener, S., Bauer, S., Krasteva, S., Vonach, C., Giessrigl, B., et al. (2010). In Vitro anti-leukemic Activity of the Ethno-Pharmacological Plant Scutellaria Orientalis Ssp. Carica Endemic to Western Turkey. Phytomedicine 17 (1), 55-62. doi:10.1016/j.phymed.2009.06.001

Pandey, V., Tripathi, A., Rani, A., and Dubey, P. K. (2020). Deoxyelephantopin, a Novel Naturally Occurring Phytochemical Impairs Growth, Induces G2/M Arrest, ROS-Mediated Apoptosis and Modulates IncRNA Expression against
Uterine Leiomyoma. Biomed. Pharmacother. 131, 110751. doi:10.1016/j.biopha. 2020.110751

Pegoraro, C. M. R., Nai, G. A., Garcia, L. A., Serra, F. M., Alves, J. A., Chagas, P. H. N., et al. (2021). Protective Effects of Bidens Pilosa on Hepatoxicity and Nephrotoxicity Induced by Carbon Tetrachloride in Rats. Drug Chem. Toxicol. 44 (1), 64-74. doi:10.1080/01480545.2018.1526182

Perera, W. H., Shivanagoudra, S. R., Pérez, J. L., Kim, D. M., Sun, Y., K Jayaprakasha, G., et al. (2021). Anti-inflammatory, Antidiabetic Properties and In Silico Modeling of Cucurbitane-type Triterpene Glycosides from Fruits of an Indian Cultivar of Momordica Charantia L. Molecules 26 (4), 1038. doi:10.3390/molecules 26041038

Poswal, F. S., Russell, G., Mackonochie, M., MacLennan, E., Adukwu, E. C., and Rolfe, V. (2019). Herbal Teas and Their Health Benefits: A Scoping Review. Plant Foods Hum. Nutr. 74 (3), 266-276. doi:10.1007/s11130-019-00750-w

Punturee, K., Wild, C. P., Kasinrerk, W., and Vinitketkumnuen, U. (2005). Immunomodulatory Activities of Centella Asiatica and Rhinacanthus Nasutus Extracts. Asian Pac. J. Cancer Prev. 6 (3), 396-400.

Puttarak, P., Charoonratana, T., and Panichayupakaranant, P. (2010). Antimicrobial Activity and Stability of Rhinacanthins-Rich Rhinacanthus Nasutus Extract. Phytomedicine 17 (5), 323-327. doi:10.1016/j.phymed.2009. 08.014

Qi, R., Li, X., Zhang, X., Huang, Y., Fei, Q., Han, Y., et al. (2020). Ethanol Extract of Elephantopus Scaber Linn. Attenuates Inflammatory Response via the Inhibition of NF-Kb Signaling by Dampening P65-DNA Binding Activity in Lipopolysaccharide-Activated Macrophages. J. Ethnopharmacol. 250, 112499. doi:10.1016/j.jep.2019.112499

Rachid, A., Rabah, D., Farid, L., Zohra, S. F., Houcine, B., and Nacéra, B. (2012). Ethnopharmacological Survey of Medicinal Plants Used in the Traditional Treatment of Diabetes Mellitus in the North Western and South Western Algeria. J. Med. Plants Res. 6 (10), 2041-2050. doi:10.13040/IJPSR.0975-8232.5(5).2006-13

Rehecho, S., Uriarte-Pueyo, I., Calvo, J., Vivas, L. A., and Calvo, M. I. (2011). Ethnopharmacological Survey of Medicinal Plants in Nor-Yauyos, a Part of the Landscape Reserve Nor-Yauyos-Cochas, Peru. J. Ethnopharmacol. 133 (1), 75-85. doi:10.1016/j.jep.2010.09.006

Rolnik, A., and Olas, B. (2021). The Plants of the Asteraceae Family as Agents in the protection of Human Health. Ijms 22 (6), 3009. doi:10.3390/ ijms 22063009

Roulette, C. J., Njau, E. A., Quinlan, M. B., Quinlan, R. J., and Call, D. R. (2018). Medicinal Foods and Beverages Among Maasai Agro-Pastoralists in Northern Tanzania. J. Ethnopharmacol 216, 191-202. doi:10.1016/j.jep.2018.01.022

Sa-Ngiamsuntorn, K., Suksatu, A., Pewkliang, Y., Thongsri, P., Kanjanasirirat, P., Manopwisedjaroen, S., et al. (2021). Anti-SARS-CoV-2 Activity of Andrographis Paniculata Extract and its Major Component Andrographolide in Human Lung Epithelial Cells and Cytotoxicity Evaluation in Major Organ Cell Representatives. J. Nat. Prod. 84 (4), 1261-1270. doi:10.1021/acs.jnatprod.0c01324

Saleem, U., Gull, Z., Saleem, A., Shah, M. A., Akhtar, M. F., Anwar, F., et al. (2021). Appraisal of Anti-parkinson Activity of Rhinacanthin-C in HaloperidolInduced Parkinsonism in Mice: a Mechanistic Approach. J. Food Biochem. 45 (4), e13677. doi:10.1111/jfbc.13677

Sánchez-Mendoza, M. E., Reyes-Ramírez, A., Cruz Antonio, L., Martínez Jiménez, L., Rodríguez-Silverio, J., and Arrieta, J. (2011). Bioassay-guided Isolation of an Anti-ulcer Compound, Tagitinin C, from Tithonia Diversifolia: Role of Nitric Oxide, Prostaglandins and Sulfhydryls. Molecules 16 (1), 665-674. doi:10.3390/ molecules 16010665

Saslis-Lagoudakis, C. H., Williamson, E. M., Savolainen, V., and Hawkins, J. A. (2011). Cross-cultural Comparison of Three Medicinal Floras and Implications for Bioprospecting Strategies. J. Ethnopharmacol 135 (2), 476-487. doi:10.1016/ j.jep.2011.03.044

Sato, Y., Suzaki, S., Nishikawa, T., Kihara, M., Shibata, H., and Higuti, T. (2000). Phytochemical Flavones Isolated from Scutellaria Barbata and Antibacterial Activity against Methicillin-Resistant Staphylococcus aureus. J. Ethnopharmacol. 72 (3), 483-488. doi:10.1016/s0378-8741(00)00265-8

Shah, M. A., Muhammad, H., Mehmood, Y., Khalil, R., Ul-Haq, Z., and Panichayupakaranant, P. (2017). Superoxide Scavenging and Antiglycation Activity of Rhinacanthins-Rich Extract Obtained from the Leaves of Rhinacanthus Nasutus. Pharmacogn. Mag. 13 (52), 652-658. doi:10.4103/pm.pm_196_17 
Shah, S. S., Shah, S. S., Iqbal, A., Ahmed, S., Khan, W. M., Hussain, S., et al. (2018). Report: Phytochemical Screening and Antimicrobial Activities of Red Silk Cotton Tree (Bombax ceiba L.). Pak. J. Pharm. Sci. 31 (3), 947-952.

Shih, K. N., Huang, W. T., Chang, C. L., and Feng, C. C. (2014). Effects of Ixeris Chinensis (Thunb.) Nakai Boiling Water Extract on Hepatitis B Viral Activity and Hepatocellular Carcinoma. Afr. J. Tradit. Complement. Altern. Med. 11 (1), 187-193. doi:10.4314/ajtcam.v1li1.30

Siripong, P., Hahnvajanawong, C., Yahuafai, J., Piyaviriyakul, S., Kanokmedhakul, K., Kongkathip, N., et al. (2009). Induction of Apoptosis by Rhinacanthone Isolated from Rhinacanthus Nasutus Roots in Human Cervical Carcinoma Cells. Biol. Pharm. Bull. 32 (7), 1251-1260. doi:10.1248/bpb.32.1251

Siripong, P., Yahuafai, J., Piyaviriyakul, S., Kanokmedhakul, K., Koide, H., Ishii, T., et al. (2012). Inhibitory Effect of Liposomal Rhinacanthin-N Isolated from Rhinacanthus Nasutus on Pulmonary Metastasis in Mice. Biol. Pharm. Bull. 35 (7), 1197-1200. doi:10.1248/bpb.b12-00244

Siripong, P., Yahuafai, J., Shimizu, K., Ichikawa, K., Yonezawa, S., Asai, T., et al. (2006). Antitumor Activity of Liposomal Naphthoquinone Esters Isolated from Thai Medicinal Plant: Rhinacanthus Nasutus KURZ. Biol. Pharm. Bull. 29 (11), 2279-2283. doi:10.1248/bpb.29.2279

Sõukand, R., and Kalle, R. (2013). Where Does the Border Lie: Locally Grown Plants Used for Making tea for Recreation And/or Healing, 1970s-1990s Estonia. J. Ethnopharmacol 150 (1), 162-174. doi:10.1016/j.jep.2013.08.031

Sõukand, R., Quave, C. L., Pieroni, A., Pardo-de-Santayana, M., Tardío, J., Kalle, R., et al. (2013). Plants Used for Making Recreational tea in Europe: a Review Based on Specific Research Sites. J. Ethnobiol. Ethnomed 9 (1), 58. doi:10.1186/1746-4269-9-58

Su, S. Y., Yang, C. H., Chiu, C. C., and Wang, Q. (2013). Acoustic Features for Identifying Constitutions in Traditional Chinese Medicine. J. Altern. Complement. Med. 19 (6), 569-576. doi:10.1089/acm.2012.0478

Sulistyani, N., and Nurkhasanah, - (2020). Screening of Anticancer, Hepatoprotective and Nephroprotective Effects of Ethanol Extract of Elephantopus Scaber L. Pak J. Pharm. Sci. 33 (3), 901-907.

Sun, C. P., Oppong, M. B., Zhao, F., Chen, L. X., and Qiu, F. (2017a). Unprecedented 22,26-seco Physalins from Physalis Angulata and Their Anti-inflammatory Potential. Org. Biomol. Chem. 15 (41), 8700-8704. doi:10.1039/c7ob02205k

Sun, C. P., Qiu, C. Y., Zhao, F., Kang, N., Chen, L. X., and Qiu, F. (2017b). Physalins V-IX, 16,24-Cyclo-13,14-Seco Withanolides from Physalis Angulata and Their Antiproliferative and Anti-inflammatory Activities. Sci. Rep. 7 (1), 4057. doi:10. 1038/s41598-017-03849-9

Sun, H. X., and Wang, H. (2006). Immunosuppressive Activity of the Ethanol Extract of Siegesbeckia Orientalis on the Immune Responses to Ovalbumin in Mice. Chem. Biodivers. 3 (7), 754-761. doi:10.1002/cbdv.200690077

Sun, P., Sun, D., and Wang, X. (2017). Effects of Scutellaria Barbata Polysaccharide on the Proliferation, Apoptosis and EMT of Human colon Cancer HT29 Cells. Carbohydr. Polym. 167, 90-96. doi:10.1016/j.carbpol.2017.03.022

Tan, F., Chen, Y., Tan, X., Ma, Y., and Peng, Y. (2017). Chinese Materia Medica Used in Medicinal Diets. J. Ethnopharmacol. 206, 40-54. doi:10.1016/j.jep.2017.05.021

Tewtrakul, S., Tansakul, P., and Panichayupakaranant, P. (2009). Anti-allergic Principles of Rhinacanthus Nasutus Leaves. Phytomedicine 16 (10), 929-934. doi:10.1016/j.phymed.2009.03.010

Thao, N. P., Binh, P. T., Luyen, N. T., Hung, T. M., Dang, N. H., and Dat, N. T. (2018). $\alpha$-Amylase and $\alpha$-Glucosidase Inhibitory Activities of Chemical Constituents from Wedelia Chinensis (Osbeck.) Merr. Leaves. J. Anal. Methods Chem. 2018, 2794904. doi:10.1155/2018/2794904

The Committee on Chinese Medicine and Pharmacy, D.o.H., Executive Yuan, Taiwan. (2003). The Catalogue of Medicinal Plant Resources in Taiwan, Taipei. 660.

The Committee on Chinese Medicine and Pharmacy, D.o.H., Executive Yuan, Taiwan. (2011). The Illustration of Common Medicinal Plants in Taiwan, Taipei. 467.

The Royal Botanic Gardens (2013). The Plant List. Available at: http://www. theplantlist.org/ (Accessed May 1, 2019).

The Royal Botanic Gardens (2021). Plants of the World Online. Available at: https://powo.science.kew.org/ (Accessed May 2021).

Toppo, E., Al-Dhabi, N. A., Sankar, C., Kumar, S. N., Buvanesvaragurunathan, K., Darvin, S. S., et al. (2021). Hepatoprotective Effect of Selected Isoandrographolide Derivatives on Steatotic HepG2 Cells and High Fat Diet Fed Rats. Eur. J. Pharmacol. 899, 174056. doi:10.1016/j.ejphar.2021.174056

Tsai, C. H., Lin, F. M., Yang, Y. C., Lee, M. T., Cha, T. L., Wu, G. J., et al. (2009). Herbal Extract of Wedelia Chinensis Attenuates Androgen Receptor Activity and Orthotopic Growth of Prostate Cancer in Nude Mice. Clin. Cancer Res. 15 (17), 5435-5444. doi:10.1158/1078-0432.CCR-09-0298

Tsai, C. H., Tzeng, S. F., Hsieh, S. C., Lin, C. Y., Tsai, C. J., Chen, Y. R., et al. (2015). Development of a Standardized and Effect-Optimized Herbal Extract of Wedelia Chinensis for Prostate Cancer. Phytomedicine 22 (3), 406-414. doi:10.1016/j.phymed.2015.01.013

Tsai, C. H., Tzeng, S. F., Hsieh, S. C., Tsai, C. J., Yang, Y. C., Tsai, M. H., et al. (2017a). A Standardized Wedelia Chinensis Extract Overcomes the Feedback Activation of HER2/3 Signaling upon Androgen-Ablation in Prostate Cancer. Front. Pharmacol. 8, 721. doi:10.3389/fphar.2017.00721

Tsai, C. H., Tzeng, S. F., Hsieh, S. C., Yang, Y. C., Hsiao, Y. W., Tsai, M. H., et al. (2017b). A Standardized Herbal Extract Mitigates Tumor Inflammation and Augments Chemotherapy Effect of Docetaxel in Prostate Cancer. Sci. Rep. 7 (1), 15624. doi:10.1038/s41598-017-15934-0

Tsai, F. J., Yang, P. Y., Chen, C. J., Li, J. P., Li, T. M., Chiou, J. S., et al. (2020). Decreased Overall Mortality Rate with Chinese Herbal Medicine Usage in Patients with Decompensated Liver Cirrhosis in Taiwan. BMC Complement. Med. Ther. 20 (1), 221. doi:10.1186/s12906-020-03010-6

Tsai, T. H., Huang, C. J., Wu, W. H., Huang, W. C., Chyuan, J. H., and Tsai, P. J. (2014). Antioxidant, Cell-Protective, and Anti-melanogenic Activities of Leaf Extracts from Wild Bitter Melon (Momordica Charantia Linn. Var. Abbreviata Ser.) Cultivars. Bot. Stud. 55 (1), 78. doi:10.1186/s40529-014-0078-y

Tsai, T. H., Huang, W. C., Ying, H. T., Kuo, Y. H., Shen, C. C., Lin, Y. K., et al. (2016). Wild Bitter Melon Leaf Extract Inhibits Porphyromonas Gingivalis-induced Inflammation: Identification of Active Compounds through Bioassay-Guided Isolation. Molecules 21 (4), 454. doi:10.3390/ molecules2 1040454

Tugume, P., and Nyakoojo, C. (2019). Ethno-pharmacological Survey of Herbal Remedies Used in the Treatment of Paediatric Diseases in Buhunga parish, Rukungiri District, Uganda. BMC Complement. Altern. Med. 19 (1), 353. doi:10. 1186/s12906-019-2763-6

Tundis, R., Rashed, K., Said, A., Menichini, F., and Loizzo, M. R. (2014). In Vitro cancer Cell Growth Inhibition and Antioxidant Activity of Bombax ceiba (Bombacaceae) Flower Extracts. Nat. Prod. Commun. 9 (5), 691-694. doi:10. $1177 / 1934578 \times 1400900527$

Ubillas, R. P., Mendez, C. D., Jolad, S. D., Luo, J., King, S. R., Carlson, T. J., et al. (2000). Antihyperglycemic Acetylenic Glucosides from Bidens Pilosa. Planta Med. 66 (1), 82-83. doi:10.1055/s-0029-1243117

Vieceli, P. S., Juiz, P. J. L., Lauria, P. S. S., Couto, R. D., Tomassini, T. C. B., Ribeiro, I. M., et al. (2021). Physalis Angulata Reduces the Progression of Chronic Experimental Periodontitis by Immunomodulatory Mechanisms. J. Ethnopharmacol. 273, 113986. doi:10.1016/j.jep.2021.113986

Visweswara Rao, P., Madhavi, K., Dhananjaya Naidu, M., and Gan, S. H. (2013a). Rhinacanthus Nasutus Ameliorates Cytosolic and Mitochondrial Enzyme Levels in Streptozotocin-Induced Diabetic Rats. Evid. Based Complement. Alternat. Med. 2013, 486047. doi:10.1155/2013/486047

Visweswara Rao, P., Madhavi, K., Dhananjaya Naidu, M., and Gan, S. H. (2013b). Rhinacanthus Nasutus Improves the Levels of Liver Carbohydrate, Protein, Glycogen, and Liver Markers in StreptozotocinInduced Diabetic Rats. Evid. Based Complement. Alternat. Med. 2013, 102901. doi:10.1155/2013/102901

Wait, S., and Chen, D. S. (2012). Towards the Eradication of Hepatitis B in Taiwan. Kaohsiung J. Med. Sci. 28 (1), 1-9. doi:10.1016/j.kjms.2011.10.027

Wang, G. K., Lin, B. B., Rao, R., Zhu, K., Qin, X. Y., Xie, G. Y., et al. (2013). A New Lignan with Anti-HBV Activity from the Roots of Bombax ceiba. Nat. Prod. Res. 27 (15), 1348-1352. doi:10.1080/14786419.2012.740032

Wang, H., Qiu, C., Chen, L., Abbasi, A. M., Guo, X., and Liu, R. H. (2019). Comparative Study of Phenolic Profiles, Antioxidant and Antiproliferative Activities in Different Vegetative Parts of Ramie (Boehmeria Nivea L.). Molecules 24 (8), 1551. doi:10.3390/molecules 24081551

Wang, H., Teng, X., Zhang, Y., Gu, Q., and He, L. (2021). Diterpenoids from the Whole Plants of Ajuga Nipponensis and Their Inhibition of RANKL-Induced Osteoclastogenesis. Chem. Biodivers. 18 (1), e2000780. doi:10.1002/cbdv. 202000780

Wang, L., Lu, S., Wang, L., Xin, M., Xu, Y., Wang, G., et al. (2021). Antiinflammatory Effects of Three Withanolides Isolated from Physalis Angulata L. In LPS-Activated RAW 264.7 Cells through Blocking NF-Kb Signaling Pathway. J. Ethnopharmacol. 276, 114186. doi:10.1016/j.jep.2021.114186 
Wang, Q., Liang, Y. Y., Li, K. W., Li, Y., Niu, F. J., Zhou, S. J., et al. (2021). Herba Siegesbeckiae: A Review on its Traditional Uses, Chemical Constituents, Pharmacological Activities and Clinical Studies. J. Ethnopharmacol. 275, 114117. doi:10.1016/j.jep.2021.114117

Wang, T. C., Lin, C. C., Lee, H. I., Yang, C., and Yang, C. C. (2010). Antihyperlipidemic Activity of Spider Brake (Pteris Multifida) with Rats Fed a High Cholesterol Diet. Pharm. Biol. 48 (2), 221-226. doi:10.3109/ 13880200903085458

Wang, T. C., Ti, M. C., Lo, S. C., and Yang, C. C. (2007). Free Radical-Scavenging Activity of Aqueous Extract of Pteris Multifida Poiret. Fitoterapia 78 (3), 248-249. doi:10.1016/j.fitote.2006.11.004

Wediasari, F., Nugroho, G. A., Fadhilah, Z., Elya, B., Setiawan, H., and Mozef, T. (2020). Hypoglycemic Effect of a Combined Andrographis Paniculata and Caesalpinia Sappan Extract in Streptozocin-Induced Diabetic Rats. Adv. Pharmacol. Pharm. Sci. 2020, 8856129. doi:10.1155/2020/8856129

Wu, D., Zhang, X., Liu, L., and Guo, Y. (2019). Key CMM Combinations in Prescriptions for Treating Mastitis and Working Mechanism Analysis Based on Network Pharmacology. Evid. Based Complement. Alternat. Med. 2019, 8245071. doi:10.1155/2019/8245071

Wu, J. N. (2005). An Illustrated Chinese Materia Medica. China: Oxiford University Press, 11p.

Wu, S. H., Hsieh, C. F., and Rejmanek, M. (2004). Catalogue of the Naturalized Flora of Taiwan. Taiwania 49 (1), 16-31. doi:10.6165/tai.2004.49(1).16

Wu, T., Wang, Q., Jiang, C., Morris-Natschke, S. L., Cui, H., Wang, Y., et al. (2015). Neoclerodane Diterpenoids from Scutellaria Barbata with Activity against Epstein-Barr Virus Lytic Replication. J. Nat. Prod. 78 (3), 500-509. doi:10.1021/np500988m

Wu, X. G., Wang, S. S., Miao, H., Cheng, J. J., Zhang, S. F., and Shang, Y. Z. (2016). Scutellaria Barbata Flavonoids Alleviate Memory Deficits and Neuronal Injuries Induced by Composited A $\beta$ in Rats. Behav. Brain Funct. 12 (1), 33. doi:10.1186/s12993-016-0118-8

Wu, Y., and Chen, D. F. (2009). Anti-complementary Effect of Polysaccharide B3PS1 in Herba Scutellariae Barbatae (Scutellaria Barbata). Immunopharmacol. Immunotoxicol. 31 (4), 696-701. doi:10.3109/08923970903095314

Wu, Y., Zhang, Z., Chen, T., Cheng, C., Zhang, Z., Zhou, H., et al. (2020). Comparison of Two Polygonum Chinense Varieties Used in Chinese Cool tea in Terms of Chemical Profiles and Antioxidant/anti-Inflammatory Activities. Food Chem. 310, 125840. doi:10.1016/j.foodchem.2019.125840

Wu, Y. H., Chiu, W. T., Young, M. J., Chang, T. H., Huang, Y. F., and Chou, C. Y. (2015). Solanum Incanum Extract Downregulates Aldehyde Dehydrogenase 1mediated Stemness and Inhibits Tumor Formation in Ovarian Cancer Cells. J. Cancer 6 (10), 1011-1019. doi:10.7150/jca.12738

Wu, Z., Yang, L., He, L., Wang, L., and Peng, L. (2020). Systematic Elucidation of the Potential Mechanisms of Core Chinese Materia Medicas in Treating Liver Cancer Based on Network Pharmacology. Evid. Based Complement. Alternat. Med. 2020, 4763675. doi:10.1155/2020/4763675

Xin, Y.-J., Choi, S., Roh, K.-B., Cho, E., Ji, H., Weon, J. B., et al. (2021). Anti-inflammatory Activity and Mechanism of Isookanin, Isolated by Bioassay-Guided Fractionation from Bidens Pilosa L. Molecules 26 (2), 255. doi:10.3390/molecules26020255

Xu, J., Chen, D., Liu, C., Wu, X. Z., Dong, C. X., and Zhou, J. (2016). Structural Characterization and Anti-tumor Effects of an Inulin-type Fructan from Atractylodes Chinensis. Int. J. Biol. Macromol. 82, 765-771. doi:10.1016/j.ijbiomac.2015.10.082

Yang, J. Y., Kim, M. G., and Lee, H. S. (2013). Acaricidal Toxicities of 1hydroxynaphthalene from Scutellaria Barbata and its Derivatives against House Dust and Storage Mites. Planta Med. 79 (11), 946-951. doi:10.1055/s-0032-1328631

Yang, W. C., Yang, C. Y., Liang, Y. C., Yang, C. W., Li, W. Q., Chung, C. Y., et al. (2019). Anti-coccidial Properties and Mechanisms of an Edible Herb, Bidens Pilosa, and its Active Compounds for Coccidiosis. Sci. Rep. 9 (1), 2896. doi:10. 1038/s41598-019-39194-2

Yang, X., Gao, X., Du, B., Zhao, F., Feng, X., Zhang, H., et al. (2018). Ilex Asprella Aqueous Extracts Exert In Vivo Anti-inflammatory Effects by Regulating the NF-Kb, JAK2/STAT3, and MAPK Signaling Pathways. J. Ethnopharmacol. 225, 234-243. doi:10.1016/j.jep.2018.06.037

Ye, C. L., and Huang, Q. (2012). Extraction of Polysaccharides from Herbal Scutellaria Barbata D. Don (Ban-Zhi-Lian) and Their Antioxidant Activity. Carbohydr. Polym. 89 (4), 1131-1137. doi:10.1016/j.carbpol.2012.03.084
Yin, M. C., Chang, C. H., Su, C. H., Yu, B., and Hsu, Y. M. (2018). Pteris Multifida, Cortex Phellodendri, and Probiotics Attenuated Inflammatory Status and Immunity in Mice with a Salmonella enterica Serovar Typhimurium Infection. Biosci. Biotechnol. Biochem. 82 (5), 836-847. doi:10.1080/ 09168451.2018 .1447356

Yu, J., Lei, J., Yu, H., Cai, X., and Zou, G. (2004). Chemical Composition and Antimicrobial Activity of the Essential Oil of Scutellaria Barbata. Phytochemistry 65 (7), 881-884. doi:10.1016/j.phytochem.2004.02.005

Yu, M. L., Chen, P. J., Dai, C. Y., Hu, T. H., Huang, C. F., Huang, Y. H., et al. (2020). 2020 Taiwan Consensus Statement on the Management of Hepatitis C: Part (I) General Population. J. Formos. Med. Assoc. 119 (6), 1019-1040. doi:10.1016/j. jfma.2020.04.003

Yu, S., Sheu, H. M., and Lee, C. H. (2017). Solanum Incanum Extract (SR-T100) Induces Melanoma Cell Apoptosis and Inhibits Established Lung Metastasis. Oncotarget 8 (61), 103509-103517. doi:10.18632/oncotarget.21508

Yuris, A., Goh, K. K. T., Hardacre, A. K., and Matia-Merino, L. (2019). The Effect of Gel Structure on the In Vitro Digestibility of Wheat Starch-Mesona Chinensis Polysaccharide Gels. Food Funct. 10 (1), 250-258. doi:10.1039/c8fo01501e

Zhang, L., Ren, B., Zhang, J., Liu, L., Liu, J., Jiang, G., et al. (2017). Anti-tumor Effect of Scutellaria Barbata D. Don Extracts on Ovarian Cancer and its Phytochemicals Characterisation. J. Ethnopharmacol. 206, 184-192. doi:10. 1016/j.jep.2017.05.032

Zhang, R., Ma, C., Wei, Y., Wang, X., Jia, J., Li, J., et al. (2021). Isolation, Purification, Structural Characteristics, Pharmacological Activities, and Combined Action of Hedyotis Diffusa Polysaccharides: A Review. Int. J. Biol. Macromol. 183, 119-131. doi:10.1016/j.ijbiomac.2021.04.139

Zhang, W., Chen, S. T., He, Q. Y., Huang, L. Q., Li, X., Lai, X. P., et al. (2018). Asprellcosides B of Ilex Asprella Inhibits Influenza A Virus Infection by Blocking the Hemagglutinin- Mediated Membrane Fusion. Front. Microbiol. 9, 3325. doi: $10.3389 /$ fmicb.2018.03325

Zhang, Y., and Li, Y. (2016). Effect of Total Flavonoids of Scutellaria Barbata on Cognitive Function and Nogo-A Expression in the hippocampus in Cerebral Ischemia Model in Gerbils. Pak. J. Pharm. Sci. 29 (6 Suppl. 1), 2373-2376.

Zhao, L. L., Makinde, E. A., Shah, M. A., Olatunji, O. J., and Panichayupakaranant, P. (2019). Rhinacanthins-rich Extract and Rhinacanthin C Ameliorate Oxidative Stress and Inflammation in Streptozotocin-Nicotinamide-Induced Diabetic Nephropathy. J. Food Biochem. 43 (4), e12812. doi:10.1111/jfbc.12812 Zhao, Z., Guo, P., and Brand, E. (2012). The Formation of Daodi Medicinal Materials. J. Ethnopharmacol 140 (3), 476-481. doi:10.1016/j.jep.2012.01.048

Zhou, X., Seto, S. W., Chang, D., Kiat, H., Razmovski-Naumovski, V., Chan, K., et al. (2016). Synergistic Effects of Chinese Herbal Medicine: A Comprehensive Review of Methodology and Current Research. Front. Pharmacol. 7, 201. doi:10. 3389/fphar.2016.00201

Zhu, H., Liang, Q. H., Xiong, X. G., Wang, Y., Zhang, Z. H., Sun, M. J., et al. (2018). Anti-Inflammatory Effects of P-Coumaric Acid, a Natural Compound of Oldenlandia Diffusa, on Arthritis Model Rats. Evid. Based Complement. Alternat. Med. 2018, 5198594. doi:10.1155/2018/5198594

Conflict of Interest: The authors declare that the research was conducted in the absence of any commercial or financial relationships that could be construed as a potential conflict of interest.

Publisher's Note: All claims expressed in this article are solely those of the authors and do not necessarily represent those of their affiliated organizations, or those of the publisher, the editors and the reviewers. Any product that may be evaluated in this article, or claim that may be made by its manufacturer, is not guaranteed or endorsed by the publisher.

Copyright $\odot 2022$ Chao, Chen, Pao, Deng, Cheng, Su and Huang. This is an openaccess article distributed under the terms of the Creative Commons Attribution License (CC BY). The use, distribution or reproduction in other forums is permitted, provided the original author(s) and the copyright owner(s) are credited and that the original publication in this journal is cited, in accordance with accepted academic practice. No use, distribution or reproduction is permitted which does not comply with these terms. 